\title{
THE INFLUENCE OF FAR-ULTRAVIOLET RADIATION ON THE PROPERTIES OF MOLECULAR CLOUDS IN THE 30 DOR REGION OF THE LARGE MAGELLANIC CLOUD
}

\author{
Jorge L. Pineda ${ }^{1,6}$, Jürgen OtT ${ }^{2,7,8}$, Ulrich Klein ${ }^{1}$, Tony Wong ${ }^{3,9,10}$, Erik Muller ${ }^{4,9,11}$, And Annie Hughes ${ }^{5,9}$ \\ ${ }^{1}$ Argelander Institut für Astronomie, Universität Bonn, Auf dem Hügel 71, D-53121 Bonn, Germany; Jorge.Pineda@jpl.nasa.gov \\ ${ }^{2}$ National Radio Astronomy Observatory, P.O. Box O, Socorro, NM 87801, USA \\ ${ }^{3}$ School of Physics, University of New South Wales, Sydney, NSW 2052, Australia \\ ${ }^{4}$ Department of Astrophysics, Nagoya University, Furo-cho, Chikusa-ku, Nagoya 464-8602, Japan \\ ${ }^{5}$ Centre for Supercomputing and Astrophysics, Swinburne University of Technology, Hawthorn, VIC 3122, Australia \\ Received 2008 December 19; accepted 2009 July 27; published 2009 September 1
}

\begin{abstract}
We present a complete ${ }^{12} \mathrm{CO} J=1 \rightarrow 0$ map of the prominent molecular ridge in the Large Magellanic Cloud (LMC) obtained with the $22 \mathrm{~m}$ ATNF Mopra Telescope. The region stretches southward by $\sim 2^{\circ}$ (or $1.7 \mathrm{kpc}$ ) from 30 Doradus, the most vigorous star-forming region in the Local Group. The location of this molecular ridge is unique insofar as it allows us to study the properties of molecular gas as a function of the ambient radiation field in a low-metallicity environment. We find that the physical properties of CO-emitting clumps within the molecular ridge do not vary with the strength of the far-ultraviolet radiation field. Since the peak $\mathrm{CO}$ brightness of the clumps shows no correlation with the radiation field strength, the observed constant value for $\mathrm{CO}-$ to- $\mathrm{H}_{2}$ conversion factor along the ridge seems to require an increase in the kinetic temperature of the molecular gas that is offset by a decrease in the angular filling factor of the $\mathrm{CO}$ emission. We find that the difference between the $\mathrm{CO}-$ to- $\mathrm{H}_{2}$ conversion factor in the molecular ridge and the outer Milky Way is smaller than has been reported by previous studies of the $\mathrm{CO}$ emission: applying the same cloud identification and analysis methods to our CO observations of the LMC molecular ridge and $\mathrm{CO}$ data from the outer Galaxy survey by Dame et al., we find that the average $\mathrm{CO}-$ to- $\mathrm{H}_{2}$ conversion factor in the molecular ridge is $X_{\mathrm{CO}} \simeq(3.9 \pm 2.5) \times 10^{20} \mathrm{~cm}^{-2}\left(\mathrm{~K} \mathrm{~km} \mathrm{~s}^{-1}\right)^{-1}$, approximately twice the value that we determine for the outer Galaxy clouds. The mass spectrum and the scaling relations between the properties of the CO clumps in the molecular ridge are similar, but not identical, to those that have been established for Galactic molecular clouds.
\end{abstract}

Key words: galaxies: ISM - ISM: molecules - ISM: structure - Magellanic Clouds

\section{INTRODUCTION}

The study of the interstellar medium (ISM) in dwarf galaxies provides important insights into the star formation history of the universe. In massive spiral galaxies, the differential rotation causes shear forces that influence the morphology and evolution of the progenitor molecular gas and the young stellar component, making the study of the their relationships difficult. In general, dwarf galaxies show little evidence of shear so we can study the relation between star formation and its prerequisites at their very locations. They may also be considered, in a hierarchical galaxy formation scenario, as local templates of the earliest galaxies. They are thought to be survivors of the merging processes that lead to the formation of larger galaxies, such as the Milky Way. Thus, the detailed study of the low-metallicity molecular gas in dwarf galaxies may help us to understand the physical conditions under which star formation took place in the main population of galaxies in the early universe. This understanding is crucial in order to interpret observations of the ISM in galaxies at high redshift.

In dwarf galaxies, stars form out of low-metallicity, low dustto-gas ratio dense molecular gas (Lisenfeld \& Ferrara 1998). Far-ultraviolet (FUV; $6 \mathrm{eV}<h v<13.6 \mathrm{eV}$ ) photons from

\footnotetext{
6 Current address: Jet Propulsion Laboratory, California Institute of Technology, 4800 Oak Grove Drive, Pasadena, CA 91109-8099, USA.

7 Also at California Institute of Technology, 1200 E. California Blvd., Caltech Astronomy, 105-24 Pasadena, CA 91125-2400, USA.

8 Jansky Fellow of the National Radio Astronomy Observatory.

9 Also at CSIRO Australia Telescope National Facility, P.O. Box 76, Epping, NSW 1710, Australia.

${ }^{10}$ Current address: Department of Astronomy, University of Illinois, Urbana, IL 61801, USA.

11 JSPS Fellow.
}

newly formed massive stars illuminate the progenitor molecular clouds producing photon-dominated regions (PDRs; e.g., 1999, and references therein). As the FUV field is mainly attenuated by dust absorption, the penetration of FUV photons is larger in low-metallicity molecular gas than in its solar metallicity counterpart. This produces an enhancement of the photodissociation rate of $\mathrm{CO}$ (increasing the relative abundance of $\mathrm{C}$ and $\mathrm{C}^{+}$) while $\mathrm{H}_{2}$ remains almost unaffected (e.g., van Dishoeck \& Black 1988). The relations between properties of the molecular gas (e.g., mass, $\mathrm{CO}$ luminosity, $\mathrm{CO}-$ to- $_{2}$ conversion factor) with metallicity and the strength of the FUV radiation field have been investigated from both theoretical (e.g., Maloney \& Black 1988; Elmegreen 1989; Sakamoto 1996) and empirical (e.g., Dettmar \& Heithausen 1989; Rubio et al. 1991; Wilson 1995; Arimoto et al. 1996; Israel 1997, 2000) perspectives. The CO-to- $\mathrm{H}_{2}$ conversion factor $\left(X_{\mathrm{CO}} \equiv N\left(\mathrm{H}_{2}\right) / I_{\mathrm{CO}}\right.$, an empirical factor that relates the intensity of $\mathrm{CO}\left[I_{\mathrm{CO}}\right]$ to the column density of $\mathrm{H}_{2}\left[N\left(\mathrm{H}_{2}\right)\right]$ ) has been found to increase for clouds located in galaxies with low metallicities and strong FUV radiation fields (Israel 1997). However, $X_{\mathrm{CO}}$ has also been shown to decrease with the FUV field by Weiß et al. (2001). This apparent contradiction arises because $X_{\mathrm{CO}}$ depends on both the metallicity and the FUV radiation field, but mutual variation of these parameters in different interstellar environments makes it difficult to determine how they each influence the value of $X_{\mathrm{CO}} \cdot{ }^{12}$

\footnotetext{
12 Note that $X_{\mathrm{CO}}$ also depends on the gas density and kinetic temperature (Maloney \& Black 1988). However, the ${ }^{12} \mathrm{CO} J=1 \rightarrow 0$ line is likely to be thermalized at the typical densities of molecular clouds and therefore its emission is not sensitive to the gas density. The kinetic temperature is related to the FUV field because the heating in the region where CO is emitted is dominated by photoelectric heating, which depends on the FUV field (e.g., Bakes \& Tielens 1994).
} 
Additional complications are introduced when low-metallicity molecular clouds are observed on different spatial scales: in particular, $X_{\mathrm{CO}}$ has been found to vary with the resolution of the observations (Rubio et al. 1993; Verter \& Hodge 1995). At higher angular resolution, the measured properties of molecular clouds in low-metallicity galaxies tend to approach those of Galactic clouds (Johansson et al. 1998; Bolatto et al. 2003; Leroy et al. 2006). Ideally, we would like to conduct high-resolution studies of molecular clouds in systems where one property of the interstellar environment varies while other environmental conditions remain constant.

The Large Magellanic Cloud (LMC) is an excellent extragalactic laboratory for such a study. Its proximity $(\sim 50 \mathrm{kpc}$; 1999, a value which we will use throughout the paper) and nearly face-on orientation $\left(i=35^{\circ}\right.$; van der Marel \& Cioni 2001) allow us to study individual clouds at high spatial resolution, while strong spatial variations in the LMC's FUV radiation field provide an opportunity to study the influence of the local FUV field strength on the properties of low-metallicity molecular gas (30\%-50\% solar; Westerlund 1997).

The most striking feature in the LMC is the 30 Doradus H II complex (hereafter 30 Dor), which is the most massive starforming region in the Local Group. Previous surveys of molecular gas in the LMC (e.g., Cohen et al. 1988; Fukui et al. 1999; Johansson et al. 1998; Kutner et al. 1997) have revealed a prominent complex of molecular clouds extending south from 30 Dor for nearly $2 \mathrm{kpc}$, which we refer to as "the 30 Dor molecular ridge." Studies of individual clouds in the 30 Dor molecular ridge have found gas densities between $\sim 10^{4}$ and $10^{5} \mathrm{~cm}^{-3}$ and kinetic temperatures between $\sim 10$ and $80 \mathrm{~K}$ (e.g., Johansson et al. 1998; Minamidani et al. 2008; Pineda et al. 2008).

The young stellar cluster R 136 located at the center of 30 Dor produces an extreme FUV radiation field. As seen in radio continuum (Dickel et al. 2005), $\mathrm{H} \alpha$ (Gaustad et al. 2001), far-infrared (FIR; Schwering 1989), and ultraviolet (e.g., Smith et al. 1987) maps, the strength of the field decreases with increasing distance from $\mathrm{R} 136$. The 30 Dor molecular ridge thus presents an ideal interstellar environment in which to investigate the properties of low-metallicity molecular gas as a continuous function of the ambient FUV radiation field.

Another aspect of interest is the global structure of the low-metallicity ISM of the LMC. Cloud structure is often characterized by the clump-mass spectrum, which describes how the total cloud mass is distributed among different-size structures. Fukui et al. (2001) presented a mass spectrum for giant molecular clouds (GMCs) in the LMC, based on NANTEN ${ }^{12} \mathrm{CO}$ data with spatial resolution of $\sim 40 \mathrm{pc}$. These authors derived a power-law index $\alpha=1.9$ for the LMC mass spectrum, which is similar to that found in the Galaxy (1.4-1.9; e.g., Elmegreen \& Falgarone 1996; Heithausen et al. 1998; Kramer et al. 1998; Williams et al. 1994). Whether this relation holds at even smaller scales, where clumps have low masses and are therefore more susceptible to the effect of enhanced $\mathrm{CO}$ photodissociation in low-metallicity environments, is still unclear. This is mainly due to the lack of wide-field, high-resolution maps of ${ }^{12} \mathrm{CO}$ emission from molecular clouds in low-metallicity environments.

In this paper, we present a complete, fully sampled, ${ }^{12} \mathrm{CO}$ $J=1 \rightarrow 0$ map of the 30 Dor molecular ridge in the LMC obtained with the ATNF Mopra $22 \mathrm{~m}$ Telescope. Parts of this region have been mapped with the SEST telescope at a similar angular resolution and in the same transition (Johansson et al. 1998; Kutner et al. 1997). Cloud properties such as sizes, CO luminosities, $X_{\mathrm{CO}}$ factor, etc., have been studied in the 30 Dor and N159/160 regions by Johansson et al. (1998) but not in the southern molecular clouds (Kutner et al. 1997), although the gravitational stability of clumps in this region has been discussed by Indebetouw et al. (2008). We combine our ${ }^{12} \mathrm{CO}$ data with a map of the strength of the FUV radiation field, derived from HIRES/IRAS $60 \mu \mathrm{m}$ and $100 \mu \mathrm{m}$ images, in order to study the dependence of the properties of low-metallicity molecular clouds on the radiation field. We also present a study of the clump-mass spectrum for the molecular clouds in the 30 Dor molecular ridge. This has not been attempted in the LMC at the resolution of our observations. The main difference between our work and that done with the SEST telescope is in the analysis of the data. Additionally, the wider coverage of our map allows us to identify clouds that have not previously been mapped at this resolution (see also Ott et al. 2008). The paper is organized as follows. The observations are summarized in Section 2. The analysis of the data is described in Section 3 and the results are discussed in Section 4. A summary is given in Section 5.

\section{OBSERVATIONS}

The ${ }^{12} \mathrm{CO} J=1 \rightarrow 0(115 \mathrm{GHz})$ observations were made using the $22 \mathrm{~m}$ ATNF Mopra ${ }^{13}$ Telescope between 2005 June and September. The ${ }^{12} \mathrm{CO} J=1 \rightarrow 0$ integrated intensity map of the 30 Dor molecular ridge is shown in Figure 1. The observational results of this map are presented elsewhere (Ott et al. 2008).

A total of 120 fields, $5^{\prime} \times 5^{\prime}$ in size, were mapped using the on-the-fly mapping mode, encompassing the entire 30 Dor molecular ridge. Typical values of the single side-band (SSB) system temperature $T_{\text {sys }}^{*}$ of about $600 \mathrm{~K}$ were obtained under clear atmospheric conditions. Prior to each map, the pointing accuracy was checked using the $\mathrm{SiO}$ maser $\mathrm{R}$ Dor, with corrections typically below $5^{\prime \prime}$.

The digital autocorrelator was configured to output 1024 channels across a $64 \mathrm{MHz}\left(163.8 \mathrm{~km} \mathrm{~s}^{-1}\right)$ band in two polarizations, corresponding to a velocity resolution of $0.16 \mathrm{~km} \mathrm{~s}^{-1}$. We used the AIPS++ task LiveData to calibrate the spectra and to remove a first-order fit, and another AIPS++ task Gridzilla to grid the data into spectral line cubes. LiveData is the processing software originally designed for data taken with the Parkes multibeam receiver and is used to apply system temperature calibration, bandpass calibration, heliocentric correction, spectral smoothing, and to write out the data in sdfits format. Gridzilla is a re-gridding software package that forms three-dimensional (R.A.-decl.-velocity) data cubes from bandpass-calibrated sdfits files (usually produced by LiveData). During the gridding procedure, the data were convolved with a Gaussian smoothing kernel with a FWHM half of the beam size $\left(33^{\prime \prime}\right.$ at $\left.115 \mathrm{GHz}\right)$, producing an effective resolution of $45^{\prime \prime}$ for the data cube. The spectra were smoothed to an angular resolution of $60^{\prime \prime}$ (corresponding to a linear scale of $\sim 15 \mathrm{pc}$ ) and to a velocity resolution of $0.96 \mathrm{~km} \mathrm{~s}^{-1}$, in order to increase the signal-to-noise ratio. The Mopra telescope has an "inner" error beam in the range of $40-80^{\prime \prime}$, which is similar to the size of emission. We therefore applied an extended beam efficiency of $\eta_{\mathrm{mb}}=0.55$ (Ladd et al. $2005)$ to convert from $T_{\mathrm{A}}^{*}$ to extended beam brightness $\left(T_{\mathrm{xb}}\right)$. The typical rms noise of the channel maps is $0.16 \mathrm{~K}$.

\footnotetext{
13 The Mopra radio telescope is part of the Australia Telescope which is funded by the Commonwealth of Australia for operation as a National Facility managed by CSIRO.
} 




Figure 1. Contour map of the integrated intensity of the ${ }^{12} \mathrm{CO} J=1 \rightarrow 0$ line observed toward the 30 Dor molecular ridge. This contour map is overlaid onto an Spitzer $24 \mu \mathrm{m}$ emission map (Meixner et al. 2006). To improve the contrast, we divide the contour map into white and black regions. The contour levels correspond to the $5 \%-85 \%$ of the peak intensity $\left(53 \mathrm{~K} \mathrm{~km} \mathrm{~s}^{-1}\right)$, in steps of $10 \%$.

In order to compare the properties of the molecular clouds located in the 30 Dor molecular ridge with Galactic clouds of approximately solar metallicity, we utilize a map of the 2nd Quadrant observed with the CfA $1.2 \mathrm{~m}$ telescope and presented by Dame et al. (2001). The 2nd Quadrant map covers the Galactic plane from $l=100^{\circ}$ to $150^{\circ}$ and $b=-2^{\circ}$ to $2^{\circ}$, with a noise level of about $0.1 \mathrm{~K}$, at a resolution of $450^{\prime \prime}$. We use this data set (kindly provided by Thomas Dame) because it samples Galactic clouds at physical scales that are similar to those of our 30 Dor molecular ridge map. A further advantage of this data is that blending of different clouds along each line of sight is negligible for outer Galaxy clouds. While the properties of molecular clouds in the outer Galaxy are known to be different to those in the inner Galaxy (e.g., May et al. 1997), another reason to compare our molecular ridge data to the outer Galaxy cloud sample is that molecular clouds in the inner Milky Way are subject to conditions that characterize the central regions of spiral galaxies (e.g., shear forces, tidal forces, strong magnetic fields, cosmic rays), and which may not be common in the LMC.

\section{ANALYSIS AND RESULTS}

\subsection{Clump Decomposition}

In order to study the properties of the molecular gas in the 30 Dor molecular ridge and compare them to properties of clouds in the outer Galaxy, we identify individual clumps in the 30 Dor molecular ridge and 2nd Quadrant data sets using the software package GAUSSCLUMPS (Stutzki \& Güsten 1990; Kramer et al. 1998). By applying the same decomposition algorithm to both data sets, we ensure that differences between the properties of molecular clouds in the two samples are not due to the decomposition algorithm. GAUSSCLUMPS decomposes the observed three-dimensional (position and velocity) intensity distribution into emission from individual clumps by fitting Gaussians to individual peaks and subtracting them in an iterative process. The iterative decomposition process is terminated when the peak intensities of a new generation of clumps fall below five times the rms noise of the original data cube. The decomposition process reports clump parameters such as their position, local standard of rest (LSR) as velocity, clump orientation, spatial FWHMs along the spatial axes $(\Delta x, \Delta y)$, brightness temperature $\left(T_{\mathrm{mb}}\right)$, and FWHM line widths $(\Delta v)$. Kramer et al. (1998) described in detail the stability of the results to variations of input parameters used in the decomposition of several molecular clouds. For the decomposition of our observations, we adopt the standard set of stiffness parameters used in their analysis.

As GAUSSCLUMPS approximates irregularly shaped features by Gaussian clumps, the algorithm can report the identification of small, low-mass clumps that are the actually the residue of previously subtracted larger structures. To reduce the impact of this effect on our analysis, we only accept Gaussian clumps with fitted sizes that are larger than $110 \%$ of the spatial resolution (i.e., the deconvolved size of the clump is at least $40 \%$ of the instrumental resolution along both spatial dimensions). The FWHM line width of a clump is limited to be larger than $2.44 \mathrm{~km} \mathrm{~s}^{-1}$ and $1.63 \mathrm{~km} \mathrm{~s}^{-1}$ for the 30 Dor molecular ridge and 2nd Quadrant data sets, respectively, i.e., we reject clumps that occupy a single pixel in velocity space. At the observed spatial scales, we do not expect that this criterion excludes a significant number of genuine clumps with small $\Delta v$. The catalog presented by Johansson et al. (1998) has only one clump with a FWHM line width less than $2.44 \mathrm{~km} \mathrm{~s}^{-1}$, even though their velocity resolution is $\sim 0.1 \mathrm{~km} \mathrm{~s}^{-1}$. Moreover, only four clumps with line widths narrower than $1.63 \mathrm{~km} \mathrm{~s}^{-1}$ are identified in the 2nd 
Quadrant (a similar number is seen in the catalog presented by May et al. 1997).

In order to determine the kinematic distances to the identified clumps in the 2nd Quadrant data set, we assume a flat Galactic rotation curve with rotation velocity $\Theta_{\mathrm{LSR}}=220 \mathrm{~km} \mathrm{~s}^{-1}$ and radius $R_{\odot}=8.5 \mathrm{kpc}$. We discarded all (194) clumps with kinematic distances lower than $2 \mathrm{kpc}$, as the estimation of kinematic distances is not accurate enough for such local emission.

We identify a total of 67 and 118 clumps in the 30 Dor molecular ridge and 2nd Quadrant data sets, respectively. The average (deconvolved) radius of the identified clumps in the 30 Dor molecular ridge and 2 nd Quadrant is $\sim 10 \mathrm{pc}$, with a dispersion of $\sim 35 \%$.

The derived properties of the identified clumps in the 30 Dor molecular ridge are listed in Table 1 . The CO luminosity of a clump is estimated using

$$
\begin{aligned}
& \frac{L_{\mathrm{CO}}}{\mathrm{K} \mathrm{km} \mathrm{s}^{-1} \mathrm{pc}^{2}}= \\
& \left(\frac{2 \pi}{8 \log (2)}\right)^{3 / 2}\left(\frac{\Delta x}{\mathrm{pc}}\right)\left(\frac{\Delta y}{\mathrm{pc}}\right)\left(\frac{\Delta v}{\mathrm{~km} \mathrm{~s}^{-1}}\right)\left(\frac{T_{\mathrm{mb}}}{\mathrm{K}}\right) .
\end{aligned}
$$

We derive the virial mass, $M_{\mathrm{vir}}$, using

$$
\frac{M_{\mathrm{vir}}}{M_{\odot}}=k\left(\frac{R}{\mathrm{pc}}\right)\left(\frac{\Delta v^{\prime}}{\mathrm{km} \mathrm{s}^{-1}}\right)^{2},
$$

where $R$ is the deconvolved clump radius and $\Delta v^{\prime}$ is the deconvolved FWHM of the observed line profile. The factor $k$ depends on the assumed density profile of a clump (MacLaren et al. 1988). In the following, we adopt a value of $k=$ 210 , corresponding to a spherical cloud with uniform density. Different values of $k$ (i.e., different density profiles) only change the scaling but not the slope of any relation involving $M_{\mathrm{vir}}$. The deconvolved clump radius is defined as

$$
\frac{R}{\mathrm{pc}}=\frac{1}{2}\left(\frac{\Delta x^{\prime}}{\mathrm{pc}}\right)^{1 / 2}\left(\frac{\Delta y^{\prime}}{\mathrm{pc}}\right)^{1 / 2},
$$

where $\Delta x^{\prime}$ and $\Delta y^{\prime}$ are the projected deconvolved FWHM angular sizes of the clump. We choose to use deconvolved values in the calculation of the virial mass because it is an intrinsic property of the cloud. Some published studies do not deconvolve the cloud parameters, and instead quote cloud property measurements that are convolved with the instrumental resolution. This leads to an overestimate of the virial mass, in particular if the cloud size and FWHM line width are of the same order as the instrumental resolution. For example, the values of $X_{\mathrm{CO}}$ obtained in our survey (see Section 3.4) are $\sim 40 \%$ larger if we use undeconvolved values for the clump radius and FWHM line width; for the 2nd Quadrant data, using undeconvolved values increases the derived $X_{\mathrm{CO}}$ values by $\sim 30 \%$. The different methods used to calculate the virial mass and other cloud parameters complicate a quantitative comparison between our results and those of previous studies.

We estimate the value of $X_{\mathrm{CO}}$ for clumps in the 30 Dor molecular ridge assuming that they are in virial equilibrium. In this case, $X_{\mathrm{CO}}$ is calculated dividing the clump's virial mass-which we assume is an accurate measure of the total mass of $\mathrm{H}_{2}$ present-by its $\mathrm{CO}$ luminosity. These quantities are the result of multiplying the peak $\mathrm{H}_{2}$ column density and $\mathrm{CO}$ intensity $\left(I_{\mathrm{CO}}\right)$ of a (Gaussian) clump by its area. Therefore,
$X_{\mathrm{CO}} \equiv N\left(\mathrm{H}_{2}\right) / I_{\mathrm{CO}}=M_{\mathrm{vir}} / L_{\mathrm{CO}}$. Note that $L_{\mathrm{CO}}$ does not need to be calculated from deconvolved values of $R$ and $\Delta v$ since $L_{\mathrm{CO}}$ is independent of the resolution of the observations.

The effect of adopting different values of $k$ in Equation (2) is to reduce $X_{\mathrm{CO}}$ by $10 \%$ and $66 \%$, for density profiles of the form $n(r) \propto r^{-1}$ and $n(r) \propto r^{-2}$, respectively. However, the relative values of $X_{\mathrm{CO}}$ in the 30 Dor Ridge and the 2nd Quadrant do not depend on the adopted density profile, as their virial masses were calculated with the same formula.

The errors in the clump properties are estimated by considering the rms noise in the positions where clumps were identified and by assuming that the clump parameters obtained by GAUSSCLUMPS $(\Delta x, \Delta y, \Delta v)$ have errors of $30 \%$. We use error propagation to obtain uncertainties for $X_{\mathrm{CO}}, M_{\mathrm{vir}}$, and $L_{\mathrm{CO}}$. The virial masses and $\mathrm{CO}$ luminosities have typically errors of $50 \%$, whereas the error of $X_{\mathrm{CO}}$ is typically $70 \%$.

We test our decomposition by comparing our measurements of the properties of clumps in the 30 Dor molecular ridge to those listed in Table 1 of Johansson et al. (1998). Johansson et al. (1998) derive cloud parameters by fitting circular Gaussians to each intensity peak in their integrated intensity maps, so an exact correspondence between the results of the two studies is not to be expected. In Figure 2, we show cumulative histograms of the FWHM line widths ${ }^{14}$ and the CO luminosities of clumps identified by us and by Johansson et al. (1998). We exclude clumps in the 30 Dor molecular ridge data set that are located outside the 30 Dor and N159/160 regions observed by Johansson et al. (1998). Furthermore, we only consider clumps identified by Johansson et al. (1998) that have similar CO luminosities to the clumps in the 30 Dor molecular ridge, as their observations are more sensitive than ours. Note that we choose $\Delta v$ and $L_{\mathrm{CO}}$ because they are independent of angular resolution which is different in the 30 Dor Ridge and Johansson et al. (1998) data sets. For our decomposition, the mean ( \pm standard deviation) $L_{\mathrm{CO}}$ is $(6.9 \pm 7.9) \times 10^{3} \mathrm{~K} \mathrm{~km} \mathrm{~s}^{-1} \mathrm{pc}^{2}$ and $\Delta v$ is $(4.9 \pm 2.1) \mathrm{km} \mathrm{s}^{-1}$. In case of Johansson et al. (1998), the mean ( \pm standard deviation) $L_{\mathrm{CO}}$ is $(6.6 \pm 8.0) \times 10^{3} \mathrm{~K} \mathrm{~km} \mathrm{~s}^{-1}$ $\mathrm{pc}^{2}$ and $\Delta v$ is $\left(5.3 \pm 1.9 \mathrm{~km} \mathrm{~s}^{-1}\right)$. Kolmogorov-Smirnov (KS) tests between the distributions of $\Delta v$ and $L_{\mathrm{CO}}$ for the two data sets indicate that the probabilities $(P)$ that the cloud samples are drawn from the same parent distribution are 0.2 and 0.1 , respectively. (We consider $P<0.05$ to be statistically significant and $P<0.1$ to be marginally significant.) The $L_{\mathrm{CO}}$ and $\Delta v$ measurements for the Johansson et al. (1998) and 30 Dor Ridge data sets cover a similar range of values, even though differences in decomposition methods used by Johansson et al. (1998) and us almost certainly affect the exact distribution of the clump properties.

In the following, we compare the results from our decomposition of the 30 Dor molecular ridge data set with those of the decomposition of the 2nd Quadrant data. As noted above, this ensures that any difference (or similarity) between these two data sets is not an effect of the adopted decomposition method.

\subsection{Estimation of the Far-ultraviolet Radiation Field}

An important feature of the 30 Dor molecular ridge is that the low-metallicity molecular gas is exposed to very different strengths of the FUV radiation field. This can be seen in maps

\footnotetext{
14 Note that we use undeconvolved values of the FWHM line width for this comparison, in order to be consistent with Johansson et al. (1998). As explained in Section 3.4, we use deconvolved values of the clump parameters in our analysis.
} 
Table 1

Clump Properties in the 30 Dor Molecular Ridge

\begin{tabular}{|c|c|c|c|c|c|c|c|c|c|c|}
\hline $\begin{array}{l}\text { Clump } \\
\text { No. }\end{array}$ & $\begin{array}{c}\alpha(\mathrm{J} 2000) \\
(\mathrm{h} \mathrm{m} \mathrm{s})\end{array}$ & $\begin{array}{c}\delta(\mathrm{J} 2000) \\
\left({ }^{\circ},{ }^{\prime \prime}\right)\end{array}$ & $\begin{array}{c}v_{\mathrm{lsr}} \\
\left(\mathrm{km} \mathrm{s}^{-1}\right)\end{array}$ & $\begin{array}{c}\Delta v^{\prime \mathrm{a}} \\
\left(\mathrm{km} \mathrm{s}^{-1}\right)\end{array}$ & $\begin{array}{c}R^{\mathrm{a}} \\
(\mathrm{pc})\end{array}$ & $\begin{array}{l}T_{\mathrm{mb}} \\
(\mathrm{K})\end{array}$ & $\begin{array}{l}\log \left(L_{\mathrm{CO}}\right)^{\mathrm{b}} \\
\left(\mathrm{K} \mathrm{km} \mathrm{s}^{-1}\right)\end{array}$ & $\begin{array}{c}\log \left(M_{\text {vir }}\right)^{\mathrm{b}} \\
\left(M_{\odot}\right)\end{array}$ & $\begin{array}{c}X_{\mathrm{CO}} \\
10^{20}\left(\mathrm{~cm}^{-2} /\left(\mathrm{K} \mathrm{km} \mathrm{s}^{-1}\right)\right)\end{array}$ & $\begin{array}{c}\chi^{\mathrm{c}} \\
{\left[\chi_{0}\right]} \\
\end{array}$ \\
\hline 1 & $05: 36: 14.455$ & $-69: 01: 48.77$ & 272.1 & $7.6 \pm 2.3$ & $7.6 \pm 1.6$ & $1.3 \pm 0.2$ & 3.7 & 5.0 & $8.1 \pm 5.8$ & 5 \\
\hline 2 & $05: 38: 30.816$ & $-69: 02: 04.52$ & 249.6 & $4.6 \pm 1.4$ & $8.7 \pm 1.8$ & $1.6 \pm 0.1$ & 3.7 & 4.6 & $3.6 \pm 2.5$ & 119 \\
\hline 3 & $05: 35: 56.511$ & $-69: 02: 17.78$ & 259.4 & $5.3 \pm 1.6$ & $10.8 \pm 2.3$ & $2.3 \pm 0.1$ & 4.0 & 4.8 & $2.8 \pm 2.0$ & 6 \\
\hline 4 & 05:38:50.957 & $-69: 03: 52.51$ & 246.7 & $6.3 \pm 1.9$ & $8.4 \pm 1.8$ & $1.6 \pm 0.1$ & 3.8 & 4.8 & $5.0 \pm 3.5$ & 407 \\
\hline 5 & $05: 35: 04.678$ & $-69: 04: 44.26$ & 256.4 & $2.9 \pm 0.9$ & $10.8 \pm 2.3$ & $2.2 \pm 0.2$ & 3.7 & 4.3 & $1.6 \pm 1.1$ & 8 \\
\hline 6 & $05: 38: 37.524$ & $-69: 07: 28.57$ & 245.7 & $2.7 \pm 0.8$ & $12.0 \pm 2.5$ & $1.0 \pm 0.1$ & 3.5 & 4.3 & $3.0 \pm 2.1$ & 318 \\
\hline 7 & $05: 39: 49.663$ & $-69: 12: 51.66$ & 244.8 & $3.0 \pm 0.9$ & $7.2 \pm 1.5$ & $1.1 \pm 0.1$ & 3.3 & 4.1 & $3.5 \pm 2.5$ & 22 \\
\hline 8 & $05: 38: 57.857$ & $-69: 15: 40.54$ & 248.7 & $3.7 \pm 1.1$ & $9.8 \pm 2.1$ & $0.8 \pm 0.1$ & 3.4 & 4.4 & $5.3 \pm 3.8$ & 36 \\
\hline 9 & $05: 40: 17.065$ & $-69: 17: 26.83$ & 282.8 & $2.8 \pm 0.8$ & $8.8 \pm 1.9$ & $0.8 \pm 0.1$ & 3.2 & 4.2 & $4.3 \pm 3.1$ & 7 \\
\hline 10 & 05:39:46.005 & $-69: 29: 03.82$ & 224.3 & $2.6 \pm 0.8$ & $9.3 \pm 2.0$ & $0.9 \pm 0.1$ & 3.2 & 4.1 & $3.3 \pm 2.4$ & 17 \\
\hline 11 & 05:39:04.934 & $-69: 29: 52.53$ & 257.4 & $3.9 \pm 1.2$ & $7.8 \pm 1.7$ & $2.0 \pm 0.1$ & 3.6 & 4.4 & $2.5 \pm 1.8$ & 81 \\
\hline 12 & $05: 38: 55.865$ & $-69: 34: 40.62$ & 247.7 & $10.4 \pm 3.1$ & $8.4 \pm 1.8$ & $1.6 \pm 0.2$ & 4.0 & 5.3 & $8.5 \pm 6.0$ & 22 \\
\hline 13 & $05: 38: 16.886$ & $-69: 34: 52.60$ & 262.3 & $4.8 \pm 1.4$ & $9.4 \pm 2.0$ & $1.6 \pm 0.2$ & 3.8 & 4.7 & $3.3 \pm 2.4$ & 12 \\
\hline 14 & $05: 39: 55.512$ & $-69: 35: 15.59$ & 228.2 & $4.0 \pm 1.2$ & $7.4 \pm 1.6$ & $1.2 \pm 0.2$ & 3.4 & 4.4 & $4.5 \pm 3.2$ & 21 \\
\hline 15 & $05: 39: 28.026$ & $-69: 36: 16.22$ & 241.8 & $5.0 \pm 1.5$ & $6.9 \pm 1.5$ & $0.8 \pm 0.1$ & 3.4 & 4.6 & $7.6 \pm 5.5$ & 32 \\
\hline 16 & $05: 39: 48.753$ & $-69: 37: 27.78$ & 229.1 & $3.2 \pm 1.0$ & $14.8 \pm 3.1$ & $1.5 \pm 0.2$ & 3.8 & 4.5 & $2.3 \pm 1.6$ & 76 \\
\hline 17 & $05: 39: 28.185$ & $-69: 40: 16.23$ & 241.8 & $4.7 \pm 1.4$ & $5.9 \pm 1.3$ & $1.0 \pm 0.1$ & 3.3 & 4.4 & $5.8 \pm 4.1$ & 51 \\
\hline 18 & $05: 39: 25.996$ & $-69: 43: 16.27$ & 237.9 & $3.1 \pm 0.9$ & $10.8 \pm 2.3$ & $1.5 \pm 0.1$ & 3.7 & 4.3 & $2.0 \pm 1.4$ & 33 \\
\hline 19 & $05: 37: 28.212$ & $-69: 44: 27.83$ & 235.0 & $3.7 \pm 1.1$ & $20.5 \pm 4.3$ & $1.0 \pm 0.1$ & 4.0 & 4.8 & $2.8 \pm 2.0$ & 10 \\
\hline 20 & $05: 40: 12.264$ & $-69: 44: 39.09$ & 233.1 & $6.3 \pm 1.9$ & $8.7 \pm 1.8$ & $3.3 \pm 0.1$ & 4.1 & 4.9 & $2.4 \pm 1.7$ & 109 \\
\hline 21 & $05: 38: 56.010$ & $-69: 44: 40.63$ & 232.1 & $11.8 \pm 3.5$ & $8.7 \pm 1.8$ & $0.9 \pm 0.1$ & 3.8 & 5.4 & $17.4 \pm 12$ & 11 \\
\hline 22 & $05: 39: 36.495$ & $-69: 45: 40.07$ & 236.9 & $6.3 \pm 1.9$ & $15.0 \pm 3.2$ & $4.6 \pm 0.2$ & 4.6 & 5.1 & $1.4 \pm 1.0$ & 173 \\
\hline 23 & $05: 37: 39.654$ & $-69: 46: 52.09$ & 237.9 & $4.0 \pm 1.2$ & $9.8 \pm 2.1$ & $1.4 \pm 0.1$ & 3.6 & 4.5 & $3.6 \pm 2.6$ & 5 \\
\hline 24 & $05: 39: 28.457$ & $-69: 47: 04.23$ & 233.1 & $4.0 \pm 1.2$ & $11.6 \pm 2.5$ & $2.1 \pm 0.1$ & 3.9 & 4.6 & $2.3 \pm 1.6$ & 47 \\
\hline 25 & $05: 39: 56.251$ & $-69: 47: 15.59$ & 233.1 & $2.4 \pm 0.7$ & $7.4 \pm 1.6$ & $1.5 \pm 0.2$ & 3.3 & 4.0 & $2.0 \pm 1.4$ & 49 \\
\hline 26 & $05: 39: 42.389$ & $-69: 47: 51.94$ & 239.9 & $5.6 \pm 1.7$ & $9.9 \pm 2.1$ & $1.8 \pm 0.2$ & 3.9 & 4.8 & $4.1 \pm 2.9$ & 36 \\
\hline 27 & $05: 37: 04.846$ & $-69: 48: 03.17$ & 251.6 & $4.3 \pm 1.3$ & $8.1 \pm 1.7$ & $2.2 \pm 0.1$ & 3.8 & 4.5 & $2.5 \pm 1.8$ & 10 \\
\hline 28 & $05: 39: 40.131$ & $-69: 49: 04.00$ & 254.5 & $9.2 \pm 2.8$ & $11.0 \pm 2.3$ & $1.0 \pm 0.2$ & 3.9 & 5.3 & $11.1 \pm 8.1$ & 13 \\
\hline 29 & $05: 39: 59.945$ & $-69: 50: 39.49$ & 235.9 & $7.6 \pm 2.3$ & $12.8 \pm 2.7$ & $4.1 \pm 0.2$ & 4.5 & 5.2 & $2.1 \pm 1.5$ & 10 \\
\hline 30 & $05: 40: 33.628$ & $-69: 50: 50.31$ & 236.9 & $5.2 \pm 1.6$ & $8.8 \pm 1.9$ & $0.8 \pm 0.1$ & 3.5 & 4.7 & $7.9 \pm 5.7$ & 12 \\
\hline 31 & $05: 39: 51.973$ & $-69: 53: 15.71$ & 237.9 & $4.5 \pm 1.3$ & $7.2 \pm 1.5$ & $1.2 \pm 0.2$ & 3.5 & 4.5 & $4.6 \pm 3.4$ & 5 \\
\hline 32 & $05: 40: 27.061$ & $-69: 55: 26.58$ & 237.9 & $3.4 \pm 1.0$ & $10.9 \pm 2.3$ & $1.1 \pm 0.2$ & 3.6 & 4.4 & $3.4 \pm 2.5$ & 3 \\
\hline 33 & $05: 39: 13.648$ & $-69: 55: 28.46$ & 234.0 & $4.5 \pm 1.3$ & $10.8 \pm 2.3$ & $1.5 \pm 0.2$ & 3.8 & 4.7 & $3.7 \pm 2.7$ & 5 \\
\hline 34 & $05: 39: 47.587$ & $-69: 58: 03.83$ & 238.9 & $2.6 \pm 0.8$ & $5.4 \pm 1.1$ & $1.2 \pm 0.2$ & 3.1 & 3.9 & $2.6 \pm 1.9$ & 4 \\
\hline 35 & $05: 40: 29.749$ & $-69: 59: 26.48$ & 235.0 & $4.7 \pm 1.4$ & $9.8 \pm 2.1$ & $1.0 \pm 0.2$ & 3.6 & 4.7 & $5.7 \pm 4.1$ & 2 \\
\hline 36 & 05:39:50.201 & $-70: 02: 51.77$ & 231.1 & $5.4 \pm 1.6$ & $7.4 \pm 1.6$ & $1.5 \pm 0.2$ & 3.6 & 4.7 & $4.9 \pm 3.5$ & 2 \\
\hline 37 & $05: 41: 10.078$ & $-70: 04: 12.62$ & 227.2 & $4.0 \pm 1.2$ & $6.3 \pm 1.3$ & $1.5 \pm 0.1$ & 3.5 & 4.3 & $3.4 \pm 2.4$ & 1 \\
\hline 38 & 05:38:06.973 & $-70: 05: 52.53$ & 248.7 & $3.9 \pm 1.2$ & $7.5 \pm 1.6$ & $0.9 \pm 0.2$ & 3.3 & 4.4 & $5.4 \pm 3.9$ & 5 \\
\hline 39 & $05: 38: 25.773$ & $-70: 05: 52.67$ & 239.9 & $4.4 \pm 1.3$ & $18.4 \pm 3.9$ & $1.8 \pm 0.2$ & 4.2 & 4.9 & $2.0 \pm 1.4$ & 5 \\
\hline 40 & $05: 39: 17.514$ & $-70: 07: 04.41$ & 236.0 & $6.2 \pm 1.9$ & $10.1 \pm 2.1$ & $2.8 \pm 0.1$ & 4.1 & 4.9 & $2.8 \pm 2.0$ & 3 \\
\hline 41 & 05:39:49.299 & $-70: 07: 33.79$ & 230.2 & $6.9 \pm 2.1$ & $11.1 \pm 2.4$ & $3.8 \pm 0.2$ & 4.4 & 5.0 & $2.2 \pm 1.6$ & 3 \\
\hline 42 & 05:39:34.007 & $-70: 07: 40.13$ & 239.9 & $3.6 \pm 1.1$ & $12.4 \pm 2.6$ & $1.4 \pm 0.1$ & 3.7 & 4.5 & $2.7 \pm 1.9$ & 3 \\
\hline 43 & 05:39:44.609 & $-70: 07: 51.91$ & 241.8 & $4.3 \pm 1.3$ & $8.4 \pm 1.8$ & $2.8 \pm 0.2$ & 3.9 & 4.5 & $1.9 \pm 1.3$ & 4 \\
\hline 44 & $05: 39: 27.011$ & $-70: 09: 16.26$ & 229.2 & $4.9 \pm 1.5$ & $8.0 \pm 1.7$ & $1.4 \pm 0.1$ & 3.6 & 4.6 & $4.6 \pm 3.3$ & 3 \\
\hline 45 & $05: 38: 39.881$ & $-70: 09: 52.69$ & 233.1 & $4.8 \pm 1.4$ & $9.3 \pm 2.0$ & $1.8 \pm 0.1$ & 3.8 & 4.6 & $3.1 \pm 2.2$ & 4 \\
\hline 46 & $05: 40: 42.497$ & $-70: 10: 01.97$ & 227.2 & $4.3 \pm 1.3$ & $17.4 \pm 3.7$ & $2.5 \pm 0.2$ & 4.3 & 4.8 & $1.6 \pm 1.1$ & 5 \\
\hline 47 & 05:41:01.407 & $-70: 10: 25.09$ & 220.4 & $2.4 \pm 0.7$ & $12.3 \pm 2.6$ & $1.2 \pm 0.2$ & 3.5 & 4.2 & $2.2 \pm 1.6$ & 2 \\
\hline 48 & 05:39:29.459 & $-70: 11: 28.22$ & 224.3 & $5.4 \pm 1.6$ & $11.1 \pm 2.4$ & $2.1 \pm 0.1$ & 4.1 & 4.8 & $2.8 \pm 2.0$ & 6 \\
\hline 49 & $05: 38: 37.524$ & $-70: 11: 52.69$ & 246.7 & $4.0 \pm 1.2$ & $12.4 \pm 2.6$ & $1.0 \pm 0.2$ & 3.6 & 4.6 & $4.5 \pm 3.3$ & 3 \\
\hline 50 & $05: 40: 51.138$ & $-70: 13: 37.60$ & 225.2 & $5.4 \pm 1.6$ & $11.0 \pm 2.3$ & $2.5 \pm 0.1$ & 4.1 & 4.8 & $2.6 \pm 1.8$ & 3 \\
\hline 51 & $05: 38: 51.720$ & $-70: 14: 16.66$ & 237.9 & $3.1 \pm 0.9$ & $13.2 \pm 2.8$ & $0.9 \pm 0.1$ & 3.6 & 4.4 & $3.3 \pm 2.4$ & 3 \\
\hline 52 & $05: 41: 06.653$ & $-70: 14: 48.84$ & 222.3 & $3.6 \pm 1.1$ & $15.3 \pm 3.2$ & $2.2 \pm 0.2$ & 4.1 & 4.6 & $1.4 \pm 1.0$ & 2 \\
\hline 53 & $05: 40: 40.816$ & $-70: 16: 50.07$ & 225.2 & $2.8 \pm 0.8$ & $17.4 \pm 3.7$ & $1.0 \pm 0.2$ & 3.7 & 4.5 & $2.5 \pm 1.8$ & 2 \\
\hline 54 & $05: 38: 42.269$ & $-70: 17: 40.69$ & 218.4 & $2.5 \pm 0.8$ & $9.1 \pm 1.9$ & $1.9 \pm 0.2$ & 3.5 & 4.1 & $1.6 \pm 1.1$ & 2 \\
\hline 55 & $05: 40: 49.285$ & $-70: 18: 25.70$ & 229.1 & $4.1 \pm 1.2$ & $18.4 \pm 3.9$ & $1.8 \pm 0.2$ & 4.2 & 4.8 & $2.1 \pm 1.5$ & 2 \\
\hline 56 & $05: 39: 15.584$ & $-70: 20: 52.45$ & 219.4 & $6.4 \pm 1.9$ & $8.8 \pm 1.9$ & $1.1 \pm 0.1$ & 3.7 & 4.9 & $7.4 \pm 5.3$ & 1 \\
\hline 57 & $05: 40: 58.143$ & $-70: 23: 13.30$ & 230.1 & $5.0 \pm 1.5$ & $19.1 \pm 4.1$ & $3.5 \pm 0.2$ & 4.6 & 5.0 & $1.2 \pm 0.8$ & 4 \\
\hline 58 & $05: 39: 44.489$ & $-70: 27: 27.94$ & 227.2 & $5.2 \pm 1.6$ & $9.3 \pm 2.0$ & $1.1 \pm 0.2$ & 3.6 & 4.7 & $5.7 \pm 4.1$ & 1 \\
\hline 59 & 05:42:05.959 & $-70: 29: 33.28$ & 230.1 & $4.3 \pm 1.3$ & $10.0 \pm 2.1$ & $1.1 \pm 0.2$ & 3.6 & 4.6 & $4.5 \pm 3.3$ & 0.3 \\
\hline 60 & $05: 40: 27.827$ & $-70: 30: 38.64$ & 227.2 & $5.6 \pm 1.7$ & $9.8 \pm 2.1$ & $1.9 \pm 0.2$ & 3.9 & 4.8 & $3.8 \pm 2.7$ & 2 \\
\hline 61 & $05: 40: 39.896$ & $-70: 31: 26.16$ & 245.7 & $3.0 \pm 0.9$ & $6.6 \pm 1.4$ & $1.4 \pm 0.2$ & 3.3 & 4.1 & $2.8 \pm 2.0$ & 2 \\
\hline 62 & $05: 40: 32.831$ & $-70: 32: 50.45$ & 224.3 & $2.8 \pm 0.8$ & $10.6 \pm 2.2$ & $0.8 \pm 0.1$ & 3.3 & 4.2 & $3.7 \pm 2.7$ & 3 \\
\hline 63 & $05: 40: 45.094$ & $-70: 35: 13.96$ & 230.1 & $2.9 \pm 0.9$ & $7.0 \pm 1.5$ & $1.0 \pm 0.2$ & 3.2 & 4.1 & $3.7 \pm 2.7$ & 2 \\
\hline 64 & $05: 41: 31.859$ & $-70: 42: 23.61$ & 230.1 & $5.6 \pm 1.7$ & $11.9 \pm 2.5$ & $1.0 \pm 0.1$ & 3.8 & 4.9 & $5.7 \pm 4.1$ & 0.3 \\
\hline 65 & $05: 40: 53.211$ & $-70: 43: 13.65$ & 235.0 & $2.5 \pm 0.8$ & $9.1 \pm 1.9$ & $1.2 \pm 0.2$ & 3.4 & 4.1 & $2.4 \pm 1.7$ & 2 \\
\hline 66 & $05: 40: 55.748$ & $-70: 44: 13.54$ & 238.9 & $5.0 \pm 1.5$ & $8.1 \pm 1.7$ & $2.9 \pm 0.2$ & 3.9 & 4.6 & $2.3 \pm 1.6$ & 2 \\
\hline 67 & $05: 41: 11.555$ & $-70: 54: 00.85$ & 234.0 & $4.1 \pm 1.2$ & $11.8 \pm 2.5$ & $1.4 \pm 0.1$ & 3.9 & 4.6 & $2.7 \pm 1.9$ & 1 \\
\hline
\end{tabular}

Notes.

${ }^{a}$ Deconvolved by the instrumental resolution.

b The CO luminosities and virial masses have typically errors of $50 \%$.

c Strength of the FUV radiation field in units of the Draine (1978) field. They have typical errors of 20\%, as this is the accuracy of the HIRAS/IRAS images used to derive this quantity (see the text). 

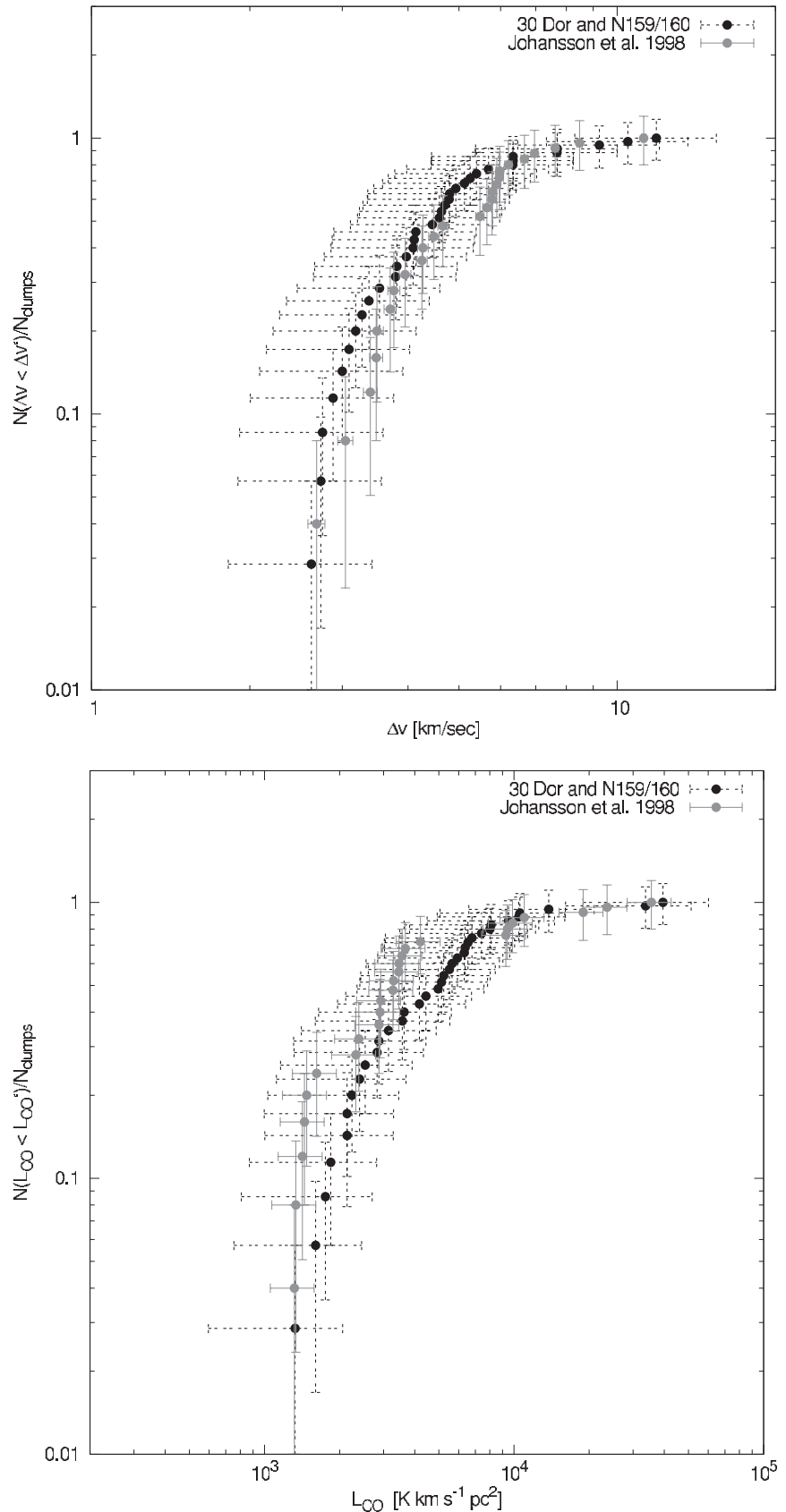

Figure 2. Cumulative histograms of the FWHM line width and CO luminosity resulting from our decomposition of the 30 Dor Ridge and the data published by Johansson et al. (1998). The FWHM line width is not deconvolved by the instrumental resolution. For all curves, the horizontal error bars reflect the uncertainties in the observed properties and the vertical error bars represent $\sqrt{N}$ counting errors.

of different tracers of the FUV radiation field, e.g., at radio continuum (Dickel et al. 2005), H $\alpha$ (Gaustad et al. 2001), FIR (Schwering 1989), and ultraviolet (e.g., Smith et al. 1987) wavelengths. For all tracers, the intensity peaks in regions that are closely associated with star formation, and declines in locations that are more distant from regions of active star formation. Assuming that metallicity gradients are negligible in the 30 Dor molecular ridge (Dufour 1984), we can therefore study clump properties as a function of the strength of the FUV radiation field. To derive a map of the strength of the FUV radiation field in the 30 Dor molecular ridge, we follow the method presented in Mookerjea et al. (2006) and Kramer et al. (2008). A map of the FIR continuum intensity ( $\left.I_{\mathrm{FIR}}\right)$ is constructed using HIRES/IRAS $100 \mu \mathrm{m}$ and $60 \mu \mathrm{m}$ images obtained from the IPAC data center. The resolution of these images has been enhanced to about $110^{\prime \prime}$ and $72^{\prime \prime}$, respectively, using a maximum correlation method (Aumann et al. 1990). The $60 \mu \mathrm{m}$ image is then convolved to $110^{\prime \prime}$, in order to match the resolution of the $100 \mu \mathrm{m}$ image. To estimate $I_{\mathrm{FIR}}$ between $42.5 \mu \mathrm{m}$ and $122.5 \mu \mathrm{m}$, we use the relationship (Helou et al. 1988; see also Nakagawa et al. 1998)

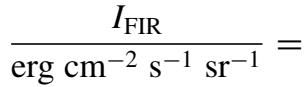

$$
\begin{aligned}
& 3.25 \times 10^{-11}\left[\frac{f_{60 \mu \mathrm{m}}}{\mathrm{Jy} \mathrm{sr}^{-1}}\right]+1.26 \times 10^{-11}\left[\frac{f_{100 \mu \mathrm{m}}}{\mathrm{Jy} \mathrm{sr}^{-1}}\right] \text {. }
\end{aligned}
$$

The dust grains in molecular clouds absorb most of the incident FUV photons, which are later re-radiated in the FIR continuum. Assuming that the contribution from other heating mechanisms such as cosmic rays can be neglected, the strength of the FUV field can be derived from $I_{\text {FIR }}$ according to

$$
\frac{\chi}{\chi_{0}}=\frac{1}{1.71} \frac{4 \pi}{\left(2 \times 1.6 \times 10^{-3}\right)} \frac{I_{\mathrm{FIR}}}{\mathrm{erg} \mathrm{cm}^{-2} \mathrm{~s}^{-1} \mathrm{sr}^{-1}}
$$

Here, $\chi$ is expressed in units of the Draine (1978) field $\left(\chi_{0}\right)$, which is related to the Habing (1968) field $\left(1.6 \times 10^{-3} \mathrm{erg} \mathrm{s}^{-1}\right.$ $\mathrm{cm}^{-2}$ ) by a factor of 1.71 when averaged over the full FUV spectrum. It is likely that the contribution of FUV photons with $h v<6 \mathrm{eV}$ increases the dust heating by a factor of $\sim 2$ (Tielens $\&$ Hollenbach 1985), and that the bolometric dust continuum intensity is a factor of $\sim 2$ larger than $I_{\mathrm{FIR}}$ (Dale et al. 2001): these two corrections approximately cancel out. Note that in the case where molecular clouds do not fill the beam, this method for estimating the strength of the FUV field provides only a lower limit.

Typical angular sizes of clumps are between $\sim 66^{\prime \prime}$ and $200^{\prime \prime}$, and as the resolution of the $\chi$ map is about $110^{\prime \prime}$, its value represents an average over an entire clump. The calibration of the $\chi$ map appears to be consistent with previous estimates of the FUV radiation field in the vicinity of the 30 Dor molecular ridge. Near the N159W cloud (Clump no. 22), for example, we obtain $\chi=173$, which is in good agreement with an independent determination from UV, radio continuum, and IRAS $60 \mu \mathrm{m}$ emission ( $\chi=120-350)$ made by Israel et al. (1996). Our estimate for $\chi$ in $\mathrm{N} 159 \mathrm{~W}$ is also consistent with the results of PDR models for this molecular cloud (e.g., Pak et al. 1998; Pineda et al. 2008). Although the $\chi=173$ may be considered low for a massive star-forming region (in the Orion Bar, for example; Walmsley et al. 2000; Young Owl et al. 2000 find $\chi \simeq 10,000$ ), Israel et al. (1996) argue that the low metallicity and low dust-to-gas ratio of this region increase the FUV photon free path lengths, resulting in a greater geometric dilution of the FUV radiation field. We stress, however, that for this analysis we are interested in the dependence of the CO clumps parameters on relative variations of the FUV radiation field.

In Figure 3, we show $\chi$ as a function of the distance to 30 Dor. Each point corresponds to the value of $\chi$ in the central position of each molecular clump identified in the 30 Dor molecular ridge. The value of $\chi$ for each clump is also listed in Table 1 . The strength of the FUV field peaks in clouds near the 30 Dor and N159 HII regions, and then decreases for more distant positions. The thick line represents the expected distribution of $\chi$ if 30 Dor and N159 were the only sources of FUV radiation, 




Figure 3. Strength of the FUV radiation field as a function of distance to 30 Dor. The values of $\chi$ are in units of the Draine 1978 field. Each data point represents the value of $\chi$ at the central position of each identified clump. The main and secondary peaks correspond to clumps close to the 30 Dor and N159 regions. The lines represent the geometrical dilution of the FUV field in case 30 Dor and N159 are considered the only sources of FUV radiation (see the text).

Table 2

Mean Values of Derived CO Clump Parameters

\begin{tabular}{lccc}
\hline \hline \multicolumn{1}{c}{ Parameter } & Mean & STDV & Units \\
\hline 30 Dor Ridge & & & \\
\hline$X_{\mathrm{CO}}$ & 3.9 & 2.5 & $10^{20} \mathrm{~cm}^{-2}\left(\mathrm{~K} \mathrm{~km} \mathrm{~s}^{-1}\right)^{-1}$ \\
$M_{\text {vir }}$ & 5.2 & 4.7 & $10^{4} M_{\odot}$ \\
$L_{\mathrm{CO}}$ & 7.6 & 8.3 & $10^{3} \mathrm{~K} \mathrm{~km} \mathrm{~s}^{-1} \mathrm{pc}^{2}$ \\
$R$ & 10 & 3 & $\mathrm{pc}$ \\
$\Delta v^{\prime}$ & 4.6 & 1.8 & $\mathrm{~km} \mathrm{~s}$ \\
$T_{\mathrm{mb}}$ & 1.7 & 0.9 & $\mathrm{~K}$ \\
\hline 2 nd Quadrant & & & $10^{20} \mathrm{~cm}^{-2}\left(\mathrm{~K} \mathrm{~km} \mathrm{~s}^{-1}\right)^{-1}$ \\
\hline$X_{\mathrm{CO}}$ & 2.0 & 1.0 & $10^{4} M_{\odot}$ \\
$M_{\text {vir }}$ & 3.6 & 3.9 & $10^{3} \mathrm{~K} \mathrm{~km} \mathrm{~s}^{-1} \mathrm{pc}^{2}$ \\
$L_{\mathrm{CO}}$ & 11 & 18 & $\mathrm{pc}$ \\
$R$ & 10 & 3.8 & $\mathrm{~km} \mathrm{~s}$ \\
$\Delta v^{\prime}$ & 3.8 & 1.4 & $\mathrm{~K}$ \\
$T_{\mathrm{mb}}$ & 3.0 & 1.6 &
\end{tabular}

where we have used the value of $\chi$ at the clump closest to the center of each $\mathrm{H}$ II region to represent the radiation source, and considering only geometrical dilution of the FUV field. The individual contribution from each of these regions is shown by a dashed line. Figure 3 shows that the radiation from 30 Dor and N159 is not sufficient to explain the observed distribution of $\chi$, and that additional OB stars along the 30 Dor Ridge must therefore contribute to the local FUV radiation field.

\subsection{Clump Properties as a Function of the FUV Radiation Field}

Figure 4 shows $L_{\mathrm{CO}}, M_{\mathrm{vir}}$, and $X_{\mathrm{CO}}$ plotted as a function of the FUV radiation field. The horizontal lines represent the mean values of the data given in Table 2 . As we can see, these quantities do not show any correlation over 3 orders of magnitude in $\chi$. To interpret the plots of $M_{\mathrm{vir}}$ and $L_{\mathrm{CO}}$ with $\chi$,
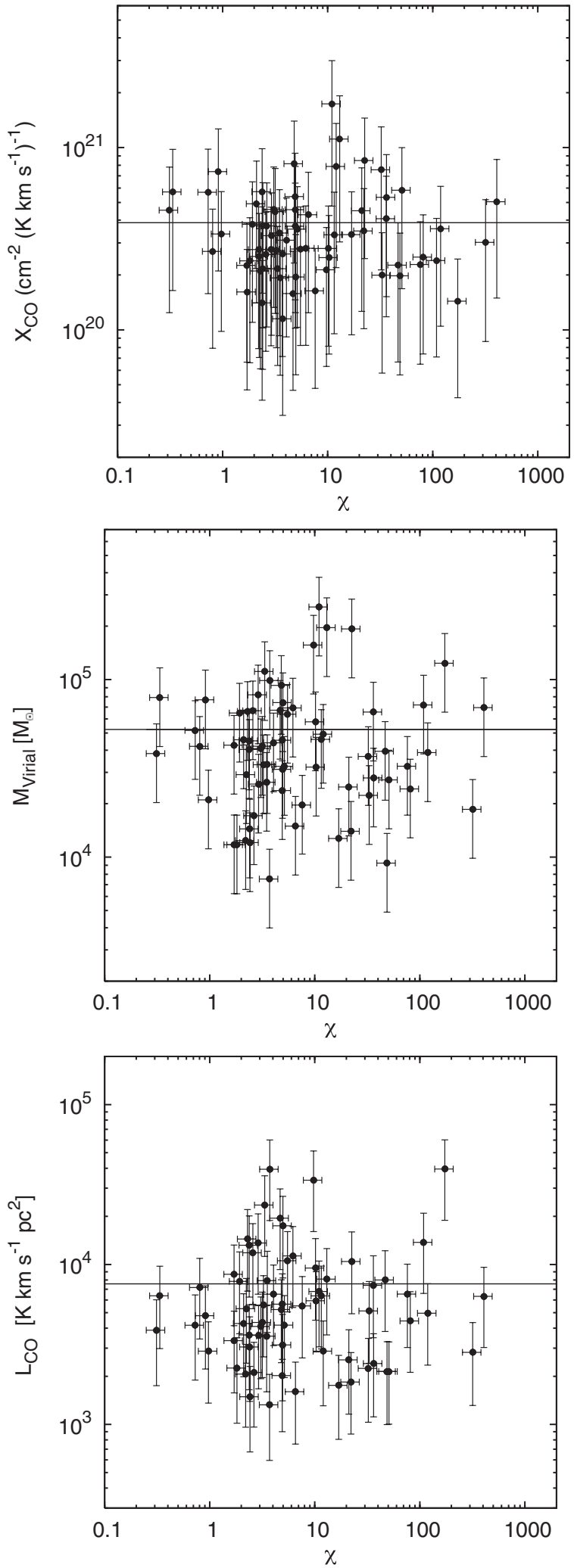

Figure 4. CO-to- $\mathrm{H}_{2}$ conversion factor $\left(X_{\mathrm{CO}}\right)$, the virial mass $\left(M_{\mathrm{vir}}\right)$, and the CO luminosity $\left(L_{\mathrm{CO}}\right)$ as functions of the strength of the FUV radiation field. The horizontal lines represent the mean values of the data (see Table 2).

we show the brightness temperature $\left(T_{\mathrm{mb}}\right)$, clump FWHM line width $\left(\Delta v^{\prime}\right)$, and clump radius $(R)$ as a function of $\chi$ in Figure 5. Again, the horizontal lines represent the mean values of the data given in Table 2. These basic clump parameters also appear to 

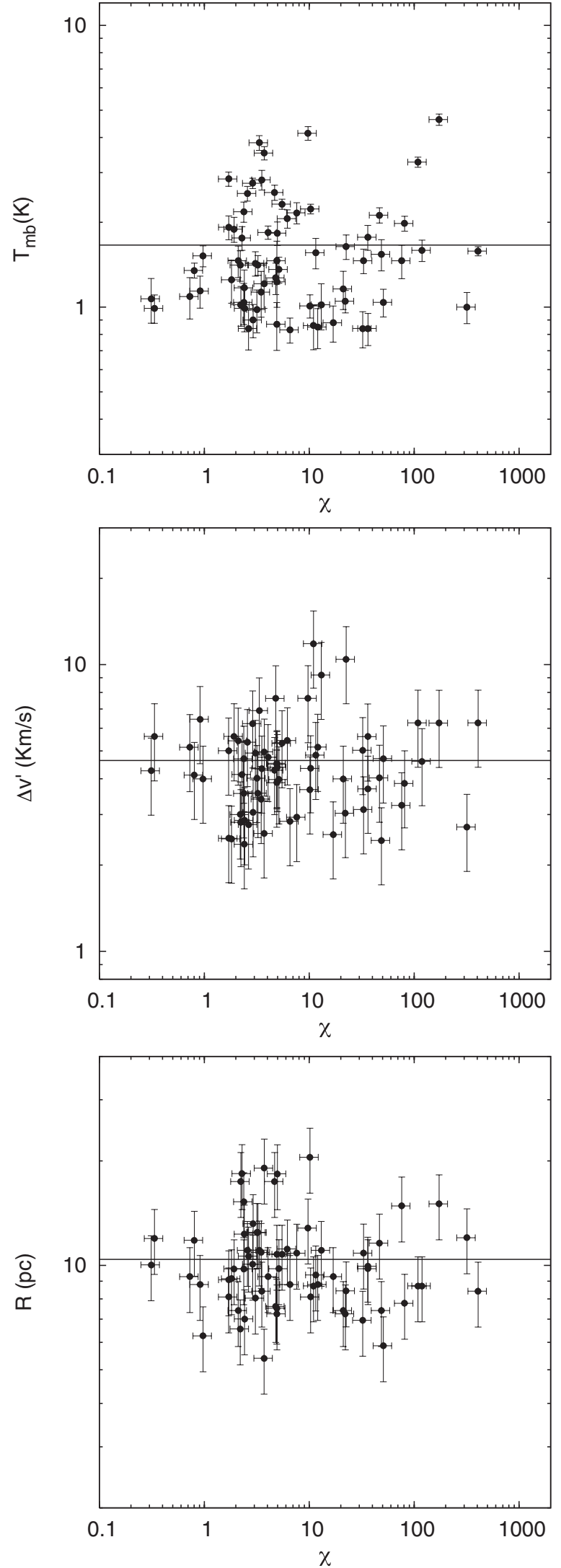

Figure 5. Brightness temperature ( $\left.T_{\mathrm{mb}}\right)$, clump FWHM line width $\left(\Delta v^{\prime}\right)$, and clump radius $(R)$ as functions of the strength of the FUV radiation field. The horizontal lines represent the mean values of the data (see Table 2)

be independent of the strength of the FUV radiation field. In Section 4 , we discuss possible reasons why the properties of the
CO clumps in the 30 Dor molecular ridge are insensitive to the strength of the FUV field.

\subsection{Comparison with Clouds in the Outer Galaxy}

We compare the properties of clumps in the 30 Dor Ridge with those of the 2nd Quadrant Galactic data. The mean values and standard deviation of these properties are listed in Table 2 and their cumulative histograms are shown in Figures 6 and 7. Although measures of their dispersion and central tendency are comparable, KS tests between the distributions of $\Delta v^{\prime}, R$, and $T_{\mathrm{mb}}$ for the two data sets indicate that the probabilities $(P)$ that the cloud samples are drawn from the same parent distribution are $0,0.07$, and 0 , respectively. These low probabilities suggest that there are differences between the distribution of CO clump properties in both regions. KS tests for the distributions of $L_{\mathrm{CO}}$, $M_{\text {vir }}$, and $X_{\mathrm{CO}}$ also result in low probabilities ( $P=0.3,0$, and $0)$, mainly due to the dissimilarity between the distribution of $\Delta v^{\prime}, T_{\mathrm{mb}}$, and $R$ in the 30 Dor Ridge and the 2nd Quadrant. In Figure 6, we can see that clumps in the 30 Dor Ridge have lower brightness temperatures compared with clumps in the 2nd Quadrant. This might be an indication that the $\mathrm{CO}$ beam filling factor is lower in the 30 Dor Ridge, which can be a result of its reduced metallicity. The 30 Dor Ridge clumps have larger FWHM line widths: 50\% of clouds in the 2nd Quadrant have FWHM line widths less than $4 \mathrm{~km} \mathrm{~s}^{-1}$ compared to only $30 \%$ in the 30 Dor Ridge. Finally, the 30 Dor Ridge also shows a deficit of clumps with $R<8$ pc compared to the 2nd Quadrant. This might be the effect of $\mathrm{CO}$ photodissociation that destroys or prevents the formation of small clumps. The differences in $\Delta v^{\prime}, T_{\mathrm{mb}}$, and $R$ result in lower CO luminosities and larger virial masses in the 30 Dor Ridge. We caution that in case of $\Delta v^{\prime}$ and $R$ the observed variations are comparable to the errors in the determinations of these quantities.

The mean value of the $X_{\mathrm{CO}}$ conversion factor in the 30 Dor molecular ridge is $\left.3.9 \pm 2.5 \times 10^{20} \mathrm{~cm}^{-2}\left(\mathrm{~K} \mathrm{~km} \mathrm{~s}^{-1}\right)^{-1}\right)$. This is almost twice the average value for $X_{\mathrm{CO}}$ in the 2nd Quadrant $\left([2.0 \pm 1.0] \times 10^{20}\left(\mathrm{~cm}^{-2} \mathrm{~K} \mathrm{~km} \mathrm{~s}^{-1}\right)^{-1}\right)$, although we note that the dispersion of $X_{\mathrm{CO}}$ values for the clumps in both data sets is quite large. Table 2 and Figure 7 show that the average virial mass of clumps in the molecular ridge is $\sim 45 \%$ larger than the average virial mass of the 2 nd Quadrant clouds, but that the average CO luminosity is $\sim 45 \%$ lower for the molecular ridge clumps. These differences account for the factor of $\sim 2$ larger value of $X_{\mathrm{CO}}$ in the 30 Dor molecular ridge. The average value of $X_{\mathrm{CO}}$ in the 30 Dor molecular ridge is consistent with the $4.3 \times 10^{20} \mathrm{~cm}^{-2}\left(\mathrm{~K} \mathrm{~km} \mathrm{~s}^{-1}\right)^{-1}$ derived by Israel et al. (2003) using SEST observations of several molecular cloud associated with $\mathrm{H}$ II regions in the LMC (not including clouds in the 30 Dor Ridge).

\subsubsection{Empirical Relations}

Several studies of molecular clouds in our Galaxy have shown that there are empirical scaling relations between the basic observable physical properties of molecular clouds (e.g., Larson 1981; Myers 1983; Dame et al. 1986; Solomon et al. 1987). These relations, often referred to as "Larson's Laws," link the FWHM line width and size of a cloud, its CO luminosity and FWHM line width, its virial mass and CO luminosity, and its CO luminosity and radius (note that only two of these relations are independent). Solomon et al. (1987), for example, found that clouds in the inner Milky Way follow power laws of the form $\Delta v \propto R^{0.5}, L_{\mathrm{CO}} \propto \Delta v^{5}, M_{\mathrm{vir}} \propto L_{\mathrm{CO}}^{0.81}$, and $L_{\mathrm{CO}} \propto R^{2.5}$. Power laws with similar slopes have already been observed for LMC 



Figure 6. Cumulative histograms of the brightness temperature $\left(T_{\mathrm{mb}}\right)$, clump FWHM line width $\left(\Delta v^{\prime}\right)$, and clump radius $(R)$ derived in the 30 Dor Ridge and 2nd Quadrant. For all curves, the horizontal error bars reflect the uncertainties in the observed properties and the vertical error bars represent $\sqrt{N}$ counting

Figure 7. Cumulative histograms of the virial mass $\left(M_{\text {vir }}\right)$, the CO luminosity $\left(L_{\mathrm{CO}}\right)$, and the CO-to- $\mathrm{H}_{2}$ conversion factor $\left(X_{\mathrm{CO}}\right)$ derived in the 30 Dor Ridge and 2nd Quadrant. For all curves, the horizontal error bars reflect the uncertainties in the observed properties and the vertical error bars represent $\sqrt{N}$ counting errors. 

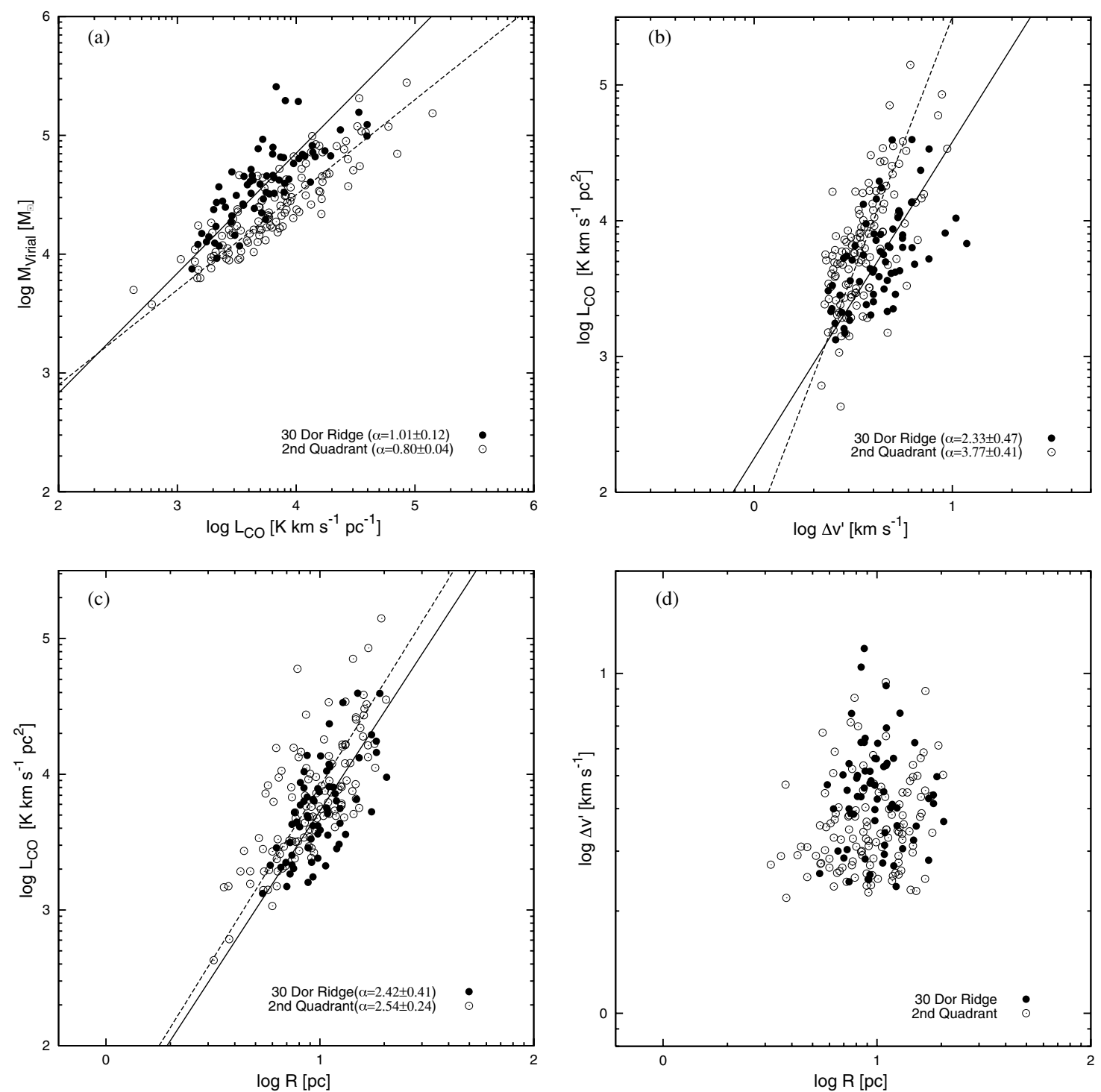

Figure 8. Virial mass-CO luminosity, CO luminosity-FWHM line width, CO luminosity-clump size, and FWHM line width-size relations for the identified clumps in the 30 Dor molecular ridge. For comparison, we also include the same relations for the clumps identified in the 2nd Quadrant. The thick and thin lines correspond to fits to the relations for the 30 Dor Ridge and 2nd Quadrant, respectively. For clarity, we do not show error bars. The typical errors in virial mass, CO luminosity, FWHM line width, and size are 50\%, 50\%, 30\%, and 20\%, respectively.

molecular clouds, with some evidence that clouds in the LMC have lower CO luminosities compared to Galactic clouds with a similar FHWM line width (e.g., Cohen et al. 1988; Garay et al. 1993; Johansson et al. 1998; Mizuno et al. 2001a). This has been previously interpreted as evidence that $\mathrm{CO}$ is significantly photodissociated in the LMC.

Figure 8 shows the observed relations between size, velocity dispersion, CO luminosity, and virial mass for the clumps identified in the 30 Dor molecular ridge and in the 2nd Quadrant. The virial mass-CO luminosity relation is shown in Figure 8(a). The orthogonal BES fit ${ }^{15}$ results in

$$
M_{\mathrm{vir}}=10^{(0.8 \pm 0.4)} L_{\mathrm{CO}}^{(1.0 \pm 0.1)}
$$

for the 30 Dor molecular ridge, and

$$
M_{\text {vir }}=10^{(1.3 \pm 0.2)} L_{\mathrm{CO}}^{(0.8 \pm 0.04)}
$$

\footnotetext{
15 We fit power laws using the orthogonal bi-variate error and intrinsic scatter method (BES; Akritas \& Bershady 1996). Available at www.astro.wisc.edu/ $\sim$ mab/archive/stats/stats.html.
}

for the 2nd Quadrant. The relation is slightly steeper for clumps in the 30 Dor molecular ridge compared to the molecular clouds in the 2nd Quadrant, such that the larger $(M>5 \times$ $10^{4} M_{\odot}$ ) clumps in the 30 Dor molecular ridge appear to be underluminous by a factor of $\sim 2.4$, relative to a 2 nd Quadrant cloud with a similar virial mass. In Section 3.5 , we will see that the number of massive clumps is larger in regions with stronger FUV radiation field $(\chi>10)$ than in regions with weaker FUV radiation field $(\chi<10)$. This suggests that effects of CO photodissociation (enhanced by the FUV radiation field) might be more significant in high-mass rather than in low-mass clumps. This might explain the apparent underluminosity of clumps with large virial masses in the 30 Dor Ridge. We note that the best-fitting virial mass-CO luminosity relation for clumps in the 30 Dor molecular ridge is linear, which implies that the value of $X_{\mathrm{CO}}\left(\propto M_{\mathrm{vir}} / L_{\mathrm{CO}}\right)$ is independent of the clump mass. In the 2nd Quadrant, by contrast, the value of $X_{\mathrm{CO}}$ decreases with increasing mass. The variation in $X_{\mathrm{CO}}$ is quite small, however, corresponding to a factor of 0.3 decrease in $X_{\mathrm{CO}}$ over roughly 2 orders of magnitude in mass. 
Figure 8(b) shows the FWHM line width-CO luminosity relation. The fits are

$$
L_{\mathrm{CO}}=10^{(2.2 \pm 0.3)} \Delta v^{\prime(2.3 \pm 0.5)}
$$

for the 30 Dor Ridge, and

$$
L_{\mathrm{CO}}=10^{(1.7 \pm 0.2)} \Delta v^{\prime(3.8 \pm 0.4)}
$$

for the 2nd Quadrant. The 30 Dor Ridge shows a shallower slope compared with that for outer Galaxy clouds. Clumps in the 30 Dor Ridge with large FWHM line widths $\left(\Delta v^{\prime}>3 \mathrm{~km}\right.$ $\left.\mathrm{s}^{-1}\right)$ have CO luminosities that are a factor of 1.4 lower than those of clumps in the 2nd Quadrant with similar $\Delta v^{\prime}$.

The CO luminosity-size relation is shown in Figure 8(c). The fits are

$$
L_{\mathrm{CO}}=10^{(1.3 \pm 0.4)} R^{(2.4 \pm 0.4)}
$$

for the 30 Dor Ridge, and

$$
L_{\mathrm{CO}}=10^{(1.4 \pm 0.2)} R^{(2.5 \pm 0.2)}
$$

for the 2nd Quadrant. Both 30 Dor Ridge and the 2nd Quadrant show a similar slope. This slope is close to $L_{\mathrm{CO}} \propto R^{2.5}$, which is expected for clouds in virial equilibrium that follow $M_{\mathrm{vir}} \propto L_{\mathrm{CO}}^{0.8}$ and $\Delta v^{\prime} \propto R^{0.5}$. The best-fitting CO luminosity-size relations that we determine imply that the mass surface density of the clumps increases slowly with increasing clump mass, although the average mass surface density for the large clumps in both data sets is in good agreement with the canonical value for molecular clouds in the inner Milky Way $\left(170 M_{\odot} \mathrm{pc}^{-2}\right.$, assuming the average $X_{\mathrm{CO}}$ values that were estimated above Solomon et al. 1987, but see Heyer et al. 2009 for evidence that this should be revised downward to $\sim 100 M_{\odot} \mathrm{pc}^{-2}$ ).

Unfortunately, we are not able to fit a power law to the FWHM line width-size relation shown in Figure 8(d), as the dynamic range in $\Delta v^{\prime}$ and $R$ is insufficient. Nevertheless, the close agreement between the mean $X_{\mathrm{CO}}$ derived from our analysis of the $\mathrm{CO}$ emission and the value determined by Dobashi et al. (2008) from dust extinction measurements suggests that the clumps in the 30 Dor molecular ridge are indeed close to virial equilibrium. As the clump properties do not show any systematic variation along the ridge (Figure 4), we conclude that the degree of virialization is uniform for all clumps (see also Indebetouw et al. 2008). Although, we see in Figure 8(d) that the clumps in the 30 Dor Ridge and the 2nd Quadrant occupy the same region in the parameter space, Table 2 and Figure 6 suggest that clumps in the 30 Dor Ridge have slightly larger $\Delta v^{\prime}$ relative to the 2nd Quadrant. This is in apparent agreement with the theory of photoionization-regulated star formation proposed by McKee (1989), which predicts that molecular clouds in low-metallicity galaxies will have larger velocity dispersions than clouds of a similar size with solar metallicity. Nonetheless, we note that this prediction assumes that the filling fraction of the $\mathrm{CO}$ emission, the densities of the CO-emitting clumps and the clump-to-cloud extinction ratios are similar for molecular clouds in different galaxies, which may not be the case (e.g., Heyer et al. 2009).

In conclusion, we find small differences between the scaling relations exhibited by the clump properties in the outer Galaxy and in the 30 Dor molecular ridge. This suggests that the basic physical properties of the molecular clumps are not especially sensitive to variations in the metallicity of the interstellar gas, or that the difference in metallicity between the 30 Dor molecular ridge and clouds in the outer Galaxy is too small to produce significant disparities between the clump properties. A similar result has been obtained across a wider range of metallicities by Bolatto et al. (2008, see also Leroy et al. 2006 and Sheth et al. 2008).

\subsection{Clump-mass Spectrum}

A general property of molecular clouds is that they show self-similar structure over a wide range of scales. This hierarchical, clumpy structure may be quantified through the clump-mass spectrum of molecular clouds, which describes how the molecular gas is distributed across low- and highmass substructures. Velocity-channel maps of molecular clouds in the Milky Way (e.g., Williams et al. 1994; Elmegreen \& Falgarone 1996; Stutzki et al. 1988; Kramer et al. 1998; Heithausen et al. 1998; Heyer \& Terebey 1998) and in external galaxies (Stanimirović et al. 2000; Stanimirović \& Lazarian 2001; Fukui et al. 2001; Kim et al. 2007) can be decomposed into clumps that exhibit a power-law mass spectrum of the form $d N / d M \propto M^{\alpha}$, where the exponent, $\alpha$, is typically observed to lie between -1.4 and -1.9 . This value of $\alpha$, which indicates that the molecular gas is preferentially distributed within massive substructures, holds over several orders of magnitude of clump mass (e.g., Heithausen et al. 1998), and is independent of the star-forming nature of the clouds (e.g., Williams et al. 1994).

In this section, we study whether the metallicity and FUV radiation field modify the distribution of clump masses in the ISM of the LMC. For this analysis, we calculate the clump-mass spectrum of the 30 Dor molecular ridge. We also calculate the mass spectrum of the clumps identified in the 2nd Quadrant, in order to compare the mass spectrum of clumps in low-metallicity and solar-metallicity gas, avoiding systematic effects produced by the selection of a particular decomposition method.

In the literature, the clump-mass spectrum based on ${ }^{12} \mathrm{CO}$ data is often calculated using clump masses derived from the clump luminosity by applying a uniform $X_{\mathrm{CO}}$ conversion factor. Since the scatter in $X_{\mathrm{CO}}$ is large (Section 3.4), in this study we prefer to use virial masses for the calculation of the mass spectrum. The total virial mass of the 30 Dor molecular ridge is about $3.5 \times 10^{6} M_{\odot}$.

We express the clump-mass spectrum in its cumulative form rather than in its differential form. This is motivated by the relatively low number of identified clumps $(N=67)$ in the 30 Dor Ridge. For such a low number of clumps, the slope of the differential clump-mass spectrum is sensitive to the binning process (which is not required by the cumulative mass spectrum). Following Rosolowsky (2005), we fit a truncated power law of the form

$$
N\left(M^{\prime}>M\right)=N_{0}\left[\left(\frac{M}{M_{0}}\right)^{\alpha+1}-1\right],
$$

to our cumulative clump-mass spectrum, where $M_{0}$ is the maximum mass of the distribution and $N_{0}$ is the number of clumps with masses larger than the mass where the distribution shows significant deviation from a power law, given by $2^{1 / \alpha+1} M_{0}$. A truncation at high masses in the cumulative mass spectrum can either be a real effect (i.e., due to a physical mechanism that regulates cloud growth) or the result of finite sampling (e.g., McKee \& Williams 1997).

We use the error-in-variables procedure described by Rosolowsky (2005) to fit a power law to cumulative mass spectra. This approach has the advantage that it accounts for both 

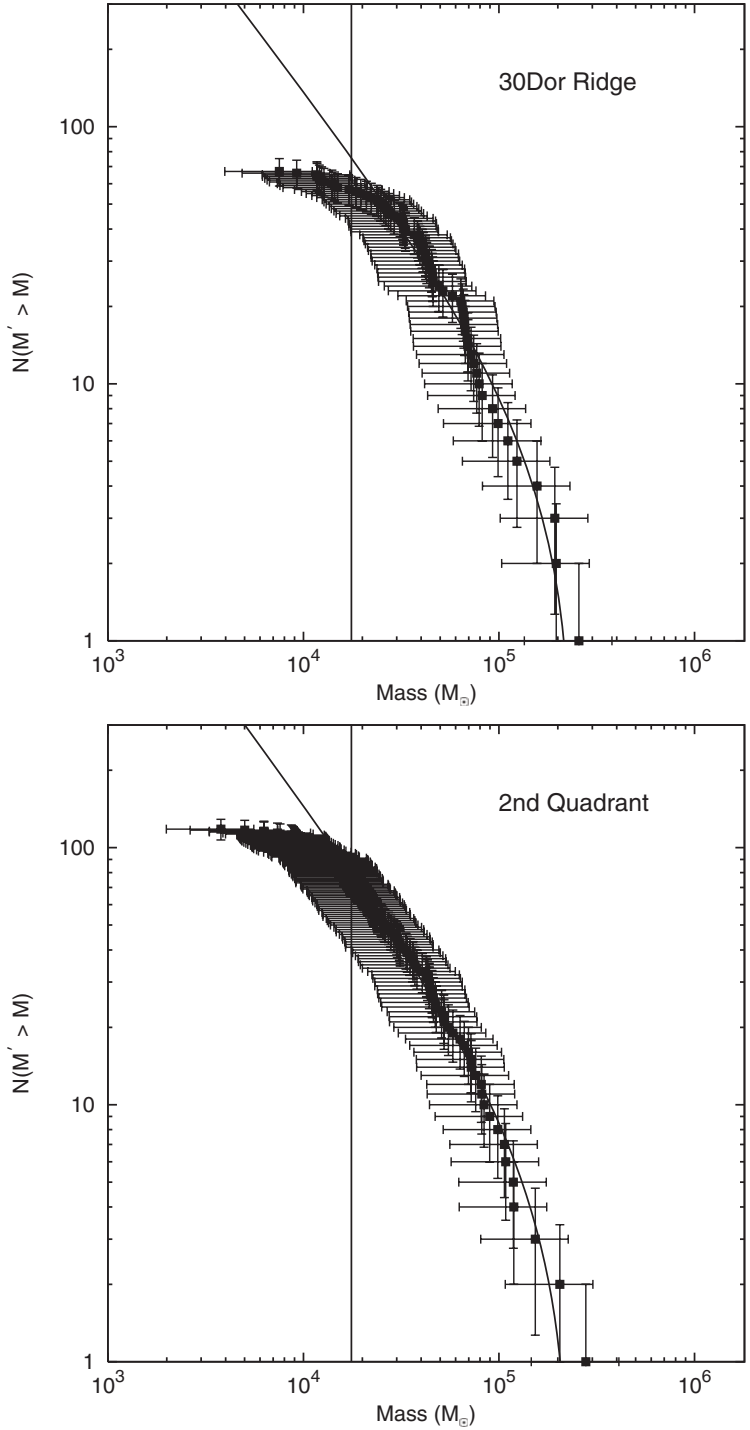

Figure 9. Cumulative clump-mass spectra for the (upper panel) 30 Dor Ridge and (lower panel) the 2nd Quadrant. The lines over the clump spectra represents the best truncated power-law fit over a range of masses above the completeness limit $\left(M>1.76 \times 10^{4} M_{\odot}\right.$; vertical line).

the $(\Delta N)^{1 / 2}$ statistical error in the distributions and the errors in the measurements of the clump masses.

The introduction of a cutoff criterion that rejects noise features and clouds with low $\mathrm{CO}$ emission in the decomposition implies that some low-mass clumps are missing from the clumpmass spectrum. This effect produces a turnover in the mass spectra at low masses. We assume that the incompleteness of our sample begins to be significant when the clump mass is lower than five times the minimum mass that can be selected in our sample based on the criteria described in Section 3.1 for the 30 Dor Ridge $\left(3.5 \times 10^{3} M_{\odot}\right)$. We therefore fit a truncated power law to the cumulative clump-mass distribution using clumps with masses greater than our adopted completeness limit of $M_{\mathrm{vir}}^{c} \simeq 2 \times 10^{4} M_{\odot}$. For consistency, we use this completeness limit for both the 30 Dor molecular ridge and the 2nd Quadrant data sets.

Figure 9 shows the cumulative clump-mass spectra of the 30 Dor molecular ridge and the 2nd Quadrant. The results of the fits are given in Table 3.

The exponents of the power-law fits to the clump-mass spectra of the 30 Dor Ridge and in the 2nd Quadrant are similar within
Table 3

Parameters of Mass Spectra for Clumps in the 30 Dor Ridge and 2nd Quadrant

\begin{tabular}{lccc}
\hline \hline \multicolumn{1}{c}{ Data Set } & $\alpha$ & $N_{0}$ & $M_{0} /\left(10^{5} M_{\odot}\right)$ \\
\hline 30 Dor Ridge & $-2.0 \pm 0.4$ & $5.9 \pm 5.9$ & $2.5 \pm 0.9$ \\
2nd Quadrant & $-2.0 \pm 0.3$ & $5.8 \pm 6.2$ & $2.4 \pm 1.1$ \\
30 Dor Ridge $(\chi>10)$ & $-1.8 \pm 0.3$ & $1.3 \pm 2.7$ & $5.5 \pm 1.6$ \\
30 Dor Ridge $(\chi<10)$ & $-1.2 \pm 0.3$ & $97 \pm 10$ & $1.2 \pm 0.3$
\end{tabular}

the errors. This suggests that the reduced metal abundance in the LMC does not significantly affect the clump-mass spectrum of molecular clouds across the observed range of clump masses. The fits are also consistent with the mass spectra that were derived by Rosolowsky (2005) for clouds in the outer Milky Way $\left(\alpha=-2.29 \pm 0.08, N_{0}=4.5 \pm 3.5\right.$, and $M_{0} / 10^{5} M_{\odot}=$ $2.9 \pm 1.0$; data from Brunt et al. 2003) and in the entire LMC $\left(\alpha=-1.71 \pm 0.19, N_{0}=10 \pm 6.5\right.$, and $M_{0} / 10^{5} M_{\odot}=$ $23 \pm 4.6$; data from Mizuno et al. 2001b) using the same fitting procedure and virial estimates for the molecular cloud masses. Note that the truncated power-law fit by Rosolowsky (2005) to the Mizuno et al. (2001b) LMC cloud catalog was based in virial mass measurements that do not account for beam deconvolution. If we fit a truncated power law to our molecular ridge data using undeconvolved measurements for the clump properties, we obtain a slope for the mass spectrum of $\alpha=-2.2 \pm 0.3$, which is still close to their results. Some of the scatter in the derived slopes is probably due to the different decomposition algorithms used to identify cloud structures in the various studies, although we note that the cloud mass spectrum constructed from the LMC cloud catalog includes clouds across the LMC, not just the molecular ridge region. It also extends to larger cloud masses and imposes a higher completeness limit than we have adopted for our analysis. Previous works that find flatter slopes for the clump-mass spectrum in the outer Galaxy (e.g., $\alpha=-1.45$; May et al. 1997) are not directly comparable to our results, as the derived slope is strongly dependent on how the mass spectrum is constructed (Rosolowsky 2005). For this reason, we prefer to compare our results with independent analyses that have employed the same representation of the mass spectrum that we have used here.

We verified that the use of virial masses instead of masses derived from $\mathrm{CO}$ luminosities and a constant value for the $X_{\mathrm{CO}}$ conversion factor does not have a significant impact on the clump-mass spectra of the 30 Dor molecular ridge and 2nd Quadrant. For clumps in the molecular ridge, assuming $X_{\mathrm{CO}}=3.9 \times 10^{20} \mathrm{~cm}^{-2}\left(\mathrm{~K} \mathrm{~km} \mathrm{~s}^{-1}\right)^{-1}$ (see Section 3.4), the bestfitting truncated power law to the clump-mass spectrum yields $\alpha=-2.2 \pm 0.3, N_{0}=1.5 \pm 2.8$, and $M_{0} / 10^{5}=2.4 \pm 0.7$. For the 2nd Quadrant, the best fit gives $\alpha=-1.9 \pm 0.2, N_{0}=5 \pm 8$, and $M_{0} / 10^{5}=4.8 \pm 2.7$, assuming $X_{\mathrm{CO}}=2.0 \times 10^{20} \mathrm{~cm}^{-2}(\mathrm{~K}$ $\left.\mathrm{km} \mathrm{s}^{-1}\right)^{-1}$. For both data sets, the fits are consistent with those determined for the cumulative mass spectra constructed from virial estimates of the clump masses.

Fukui et al. (2001) obtain a slope for the molecular cloud mass spectrum in the entire LMC of -1.9 , covering a range of cloud masses from $\sim 8 \times 10^{4}$ to $3 \times 10^{6} M_{\odot}$. Our analysis shows that the power-law relation extends to even lower masses $\left(\sim 2 \times 10^{4} M_{\odot}\right)$, suggesting that the ISM in the LMC might show the self-similarity over a wide range of scales, as observed for molecular clouds in the Milky Way (Heithausen et al. 1998).

In order to study the influence of the strength of the FUV radiation field on the clump-mass spectrum, we divide the clumps in the 30 Dor molecular ridge into low-FUV $(\chi<10)$ 

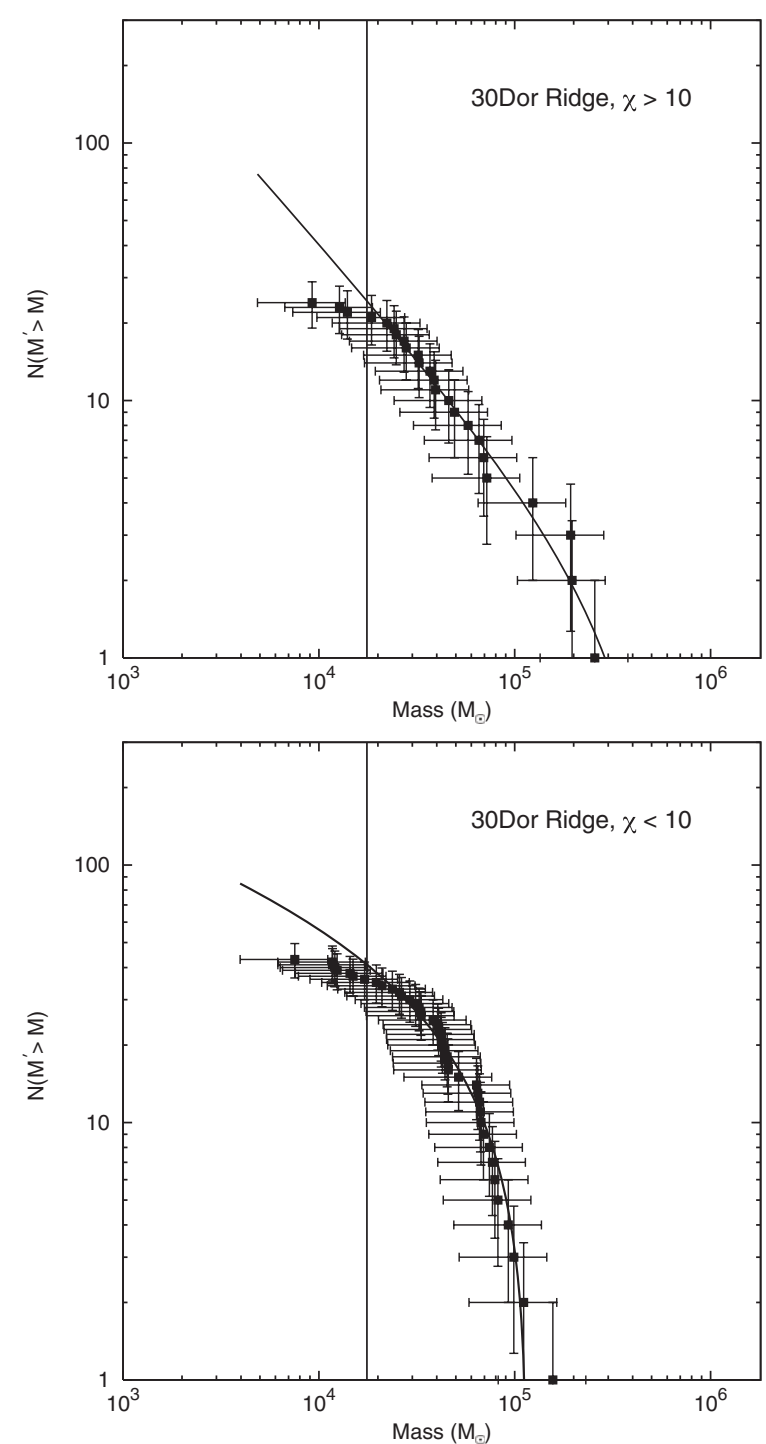

Figure 10. Cumulative clump-mass spectra for the 30 Dor Ridge for clumps with $\chi>10$ (upper panel) and with $\chi<10$ (lower panel). The lines over the clump spectra represent the best truncated power-law fit over a range of masses above the completeness limit ( $M \gtrsim 2 \times 10^{4} M_{\odot}$; vertical line).

and high-FUV $(\chi>10)$ subsamples. The resulting clump-mass spectra are shown in Figure 10 and the results of the truncated power-law fits to the spectra are listed in Table 3. The derived slope for the $\chi<10$ subsample $(-1.2 \pm 0.3)$ is dominated by low-mass clumps, and the mass spectrum steepens considerably for larger clump masses. If we fit the spectrum with a simple power law (i.e., without truncation) of the form

$$
N\left(M^{\prime}>M\right)=\left(\frac{M}{M_{0}}\right)^{\alpha+1}
$$

where $M_{0}$ and $\alpha$ represent the same quantities used in Equation (12), we obtain a much steeper slope of $\alpha=-2.7 \pm$ 0.2 . Figure 10 shows that there is a deficit of high-mass clumps with $\chi<10$. This might be because massive stars form preferentially in massive rather than in low-mass clumps. Since the strength of the local FUV field depends on embedded massive stars, the average FUV field will be larger for massive clumps than for low-mass clumps, leading to the apparent deficit of large clumps with $\chi<10$.

\section{DISCUSSION}

As outlined in the previous sections, the properties of molecular clouds in the 30 Dor molecular ridge, as traced by their $\mathrm{CO}$ emission, are not significantly affected by the varying intensity of the FUV radiation field interacting with the reducedmetallicity gas of the LMC. The requisite physical conditions for $\mathrm{CO}$ emission may provide a possible explanation for this lack of dependence. As the photodissociation of $\mathrm{CO}$ is enhanced in low-metallicity systems, $\mathrm{CO}$ emission is only present in regions with large $\mathrm{H}_{2}$ column densities. Thus, $\mathrm{CO}$ observations might only trace regions that are well shielded from the FUV radiation, as suggested by, e.g., Johansson et al. (1998). In this case, the 30 Dor molecular ridge might contain a substantial fraction of $\mathrm{H}_{2}$ coexisting with other carbon species such as $\mathrm{C}$ and $\mathrm{C}^{+}$, and this "CO-dark" fraction of the molecular gas may be larger than for clouds in the Milky Way (Grenier et al. 2005; $10 \%-50 \%$ ). Indeed, Pineda et al. (2008) recently found that the column density of CO toward LMC N159W is very much reduced compared to the column densities of $\mathrm{C}$ and $\mathrm{C}+$. This might be a reason why studies of molecular clouds in low-metallicity galaxies based on dust emission (which should trace the entire molecular content of clouds once the contribution from $\mathrm{HI}$ is subtracted) usually measure larger molecular masses than studies based on CO emission (e.g., Israel 1997; Rubio et al. 2004; Leroy et al. 2007; Bot et al. 2007). However, the measurement of molecular gas masses from dust emission usually assumes that the dust and gas are well mixed, and further relies on estimates of the dust-to-gas ratio, dust temperature, and optical properties of dust grains. Using near infrared extinction, a method that is independent of the cloud's dynamical state and of cloud decomposition method, Dobashi et al. (2008) obtained an average $X_{\mathrm{CO}}$ conversion factor of $2.26 \times 10^{20} \mathrm{~cm}^{-2}\left(\mathrm{~K} \mathrm{~km} \mathrm{~s}^{-1}\right)^{-1}$ in the entire LMC which is a factor of $\sim 1.7$ lower than our mean value of the 30 Dor Ridge. ${ }^{16}$ Note that the Dobashi et al. (2008) value refers to an $\mathrm{H}_{2}$ column density associated with $\mathrm{CO}$-emitting gas.

Elmegreen (1989) modeled the relation between $X_{\mathrm{CO}}$ and the strength of the FUV field as $X_{\mathrm{CO}} \propto \chi^{3 / 8} / T$, where $T$ is the ${ }^{12} \mathrm{CO} J=1 \rightarrow 0$ brightness temperature. We can test this prediction using our data (see Figure 11). Note that the relation presented by Elmegreen (1989) also depends weakly on the clump mass and on the ambient pressure, although we ignore their contribution here. We corrected the observed ${ }^{12} \mathrm{CO}$ $J=1 \rightarrow 0$ peak brightness temperature assuming a beam filling factor of $1 / 6$ (Pineda et al. 2008). Figure 11 shows that our data are inconsistent $\left(\chi^{2} \simeq 254\right)$ with the theoretical prediction by Elmegreen (1989). Our result that $X_{\mathrm{CO}}$ is not correlated with $\chi$ for the molecular clumps in our survey also appears to contradict the $X_{\mathrm{CO}} \propto \chi$ relation obtained by Israel (1997). However, the range of FUV radiation field estimates in that paper (which are derived from infrared observations and expressed in terms of the radiative energy per nucleon) is quite limited, whereas our estimate of $\chi$ extends over about 3 orders of magnitude. We also note that the cloud mass estimates from Israel (1997) are based on dust observations. If $X_{\mathrm{CO}}$ is independent of $\chi$ for $\mathrm{CO}$ observations and $X_{\mathrm{CO}} \propto \chi$ for dust observations, this would imply that the amount of "CO-dark" molecular gas increases with the strength of the FUV radiation field. However, this would seem to be in contradiction with

\footnotetext{
16 The value of $X_{\mathrm{CO}}$ derived from dust extinction depends on the adopted extinction to total column density ratio. When using the values adopted by Imara \& Blitz (2007), their $X_{\mathrm{CO}}$ factor is $3.55 \times 10^{20} \mathrm{~cm}^{-2}\left(\mathrm{~K} \mathrm{~km} \mathrm{~s}^{-1}\right)^{-1}$, in closer agreement with our determination.
} 


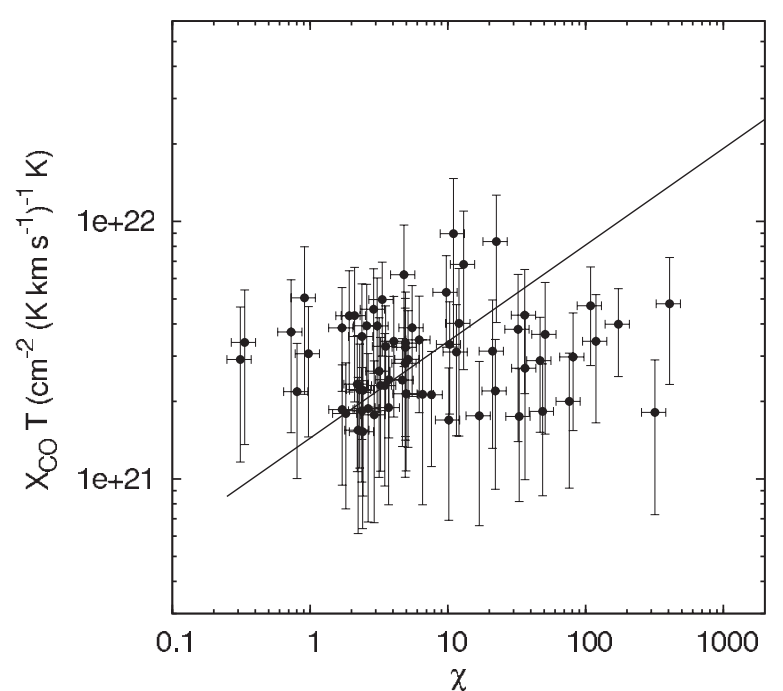

Figure 11. Relation between $X_{\mathrm{CO}}, T$, and the strength of the FUV radiation field for the clumps in the 30 Dor Ridge data set (solid black boxes). The straight line is the relation predicted by Elmegreen (1989).

the results of Dobashi et al. (2008), who found no systematic variation of the extinction-based $X_{\mathrm{CO}}$ as a function of distance to 30 Doradus (i.e., from quiescent regions to regions exposed to strong FUV radiation fields).

The observed relation between $X_{\mathrm{CO}}$ and $\chi$ can be reconciled with the theory of Elmegreen (1989) if the kinetic temperature scales with the FUV radiation field. Since the CO brightness temperature does not show significant variations with $\chi$ (Figure 5), this would require that the beam filling decreases with $\chi$ throughout the 30 Dor Ridge. It is plausible that the kinetic is larger in regions with higher $\chi$, since photoelectric heating is likely to be the dominant heating mechanism. For example, if clouds with $\chi=1$ have $T_{\text {kin }}=10 \mathrm{~K}$, kinetic temperatures of $32 \mathrm{~K}$ and $66 \mathrm{~K}$ are required for N159W $(\chi=173)$ and 30 Dor$10(\chi=407)$, respectively, to compensate any increase of $X_{\mathrm{CO}}$ with $\chi$. These kinetic temperatures are consistent with estimations in the literature (e.g., Johansson et al. 1998; Minamidani et al. 2008; Pineda et al. 2008). A systematic decrease of the beam filling with $\chi$ might be the result of an enhanced rate of $\mathrm{CO}$ photodissociation which reduces the size of $\mathrm{CO}$ cores within the beam. However, other mechanism might also play a role, e.g., cloud disruption by stellar winds from newly formed stars, etc.

The average $X_{\mathrm{CO}}$ conversion factor found in our survey of the 30 Dor molecular ridge is a factor of $\sim 2$ larger than that found in outer Galaxy clouds, a difference that is smaller compared with previous studies of clouds in the LMC at coarser angular resolutions. As we can see in Figure 1, the molecular ridge is composed of a few cloud complexes with linear scales between 100 and $300 \mathrm{pc}$. These cloud complexes show substructure characterized by compact clumps connected by low-surface brightness gas on scales between 10 and $30 \mathrm{pc}$. Since GAUSSCLUMPS decomposes the $\mathrm{CO}$ emission and identifies clumps by fitting local CO intensity peaks, this substructure is the one characterized in the present paper. Therefore, the average $X_{\mathrm{CO}}$ estimated in our survey refers to scales between 10 and $30 \mathrm{pc}$. The large cloud complexes do not necessarily have the same $X_{\mathrm{CO}}$ as the individual clumps. Although increases in the clump size are tracked by increasing estimates for the virial mass, the $\mathrm{CO}$ luminosity will not show an equivalent increase if a significant quantity of low-surface brightness interclump gas is present. In this case,

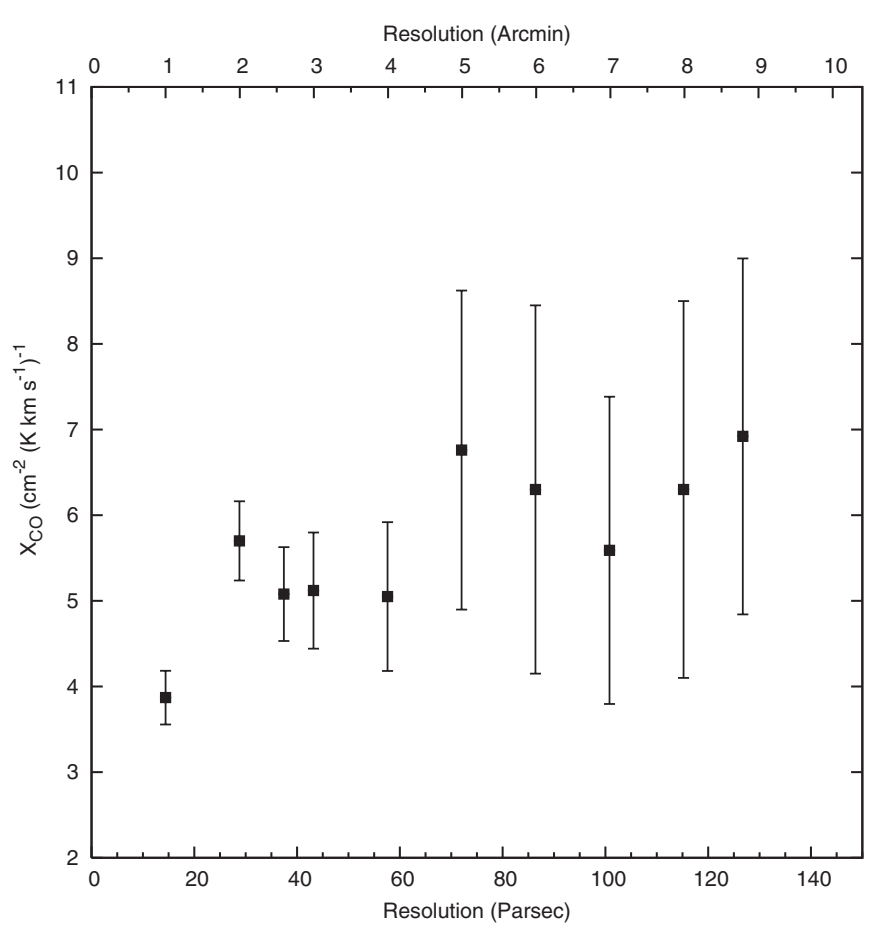

Figure 12. Average $X_{\mathrm{CO}}$ obtained for the 30 Dor Ridge map for different angular resolutions. The error bars correspond to the standard error of the mean.

the appropriate $X_{\mathrm{CO}}$ conversion factor will be higher for larger scale molecular gas structures, as shown by, e.g., Bolatto et al. (2003). Observations at coarser angular resolution trace these large-scale complexes rather than small clumps, which helps to explain the larger values of $X_{\mathrm{CO}}$ derived by previous surveys in the LMC. An example of how $X_{\mathrm{CO}}$ increases with the resolution of the observations is shown in Figure 12, where we plot the average $X_{\mathrm{CO}}$ obtained when GAUSSCLUMPS is applied to versions of the 30 Dor molecular ridge data cube that have been smoothed to different angular resolutions. We include the average $X_{\mathrm{CO}}$ for $2.6(40 \mathrm{pc})$ and 8.8 (127 pc), corresponding to the resolution of the NANTEN (Fukui et al. 2008) and CfA $1.2 \mathrm{~m}$ (Cohen et al. 1988 ) telescopes. At the resolution of the NANTEN survey, we obtain an average value of $X_{\mathrm{CO}} \sim 5.1 \times 10^{20} \mathrm{~cm}^{-2}\left(\mathrm{~K} \mathrm{~km} \mathrm{~s}^{-1}\right)^{-1}$, which is somewhat lower than the value obtained by Fukui et al. (2008), $X_{\mathrm{CO}}=7 \times 10^{20} \mathrm{~cm}^{-2}\left(\mathrm{~K} \mathrm{~km} \mathrm{~s}^{-1}\right)^{-1}$. Our result is in better agreement with the determination by Blitz et al. (2007) for the same data set, $X_{\mathrm{CO}}=5.4 \times 10^{20} \mathrm{~cm}^{-2}\left(\mathrm{~K} \mathrm{~km} \mathrm{~s}^{-1}\right)^{-1}$ : the discrepancy between the Blitz et al. (2007) and Fukui et al. (2008) may arise from a different choice of input parameters for the cloud decomposition algorithm (CLOUDPROPS; Rosolowsky \& Leroy 2006). At the resolution of the Cohen et al. (1988) observations, we obtain $X_{\mathrm{CO}} \simeq 6.9 \times 10^{20} \mathrm{~cm}^{-2}\left(\mathrm{~K} \mathrm{~km} \mathrm{~s}^{-1}\right)^{-1}$, a factor of $\sim 1.6$ lower than the value quoted by Cohen et al. (1988) $\left(X_{\mathrm{CO}} \simeq 11 \times 10^{20} \mathrm{~cm}^{-2}\left(\mathrm{~K} \mathrm{~km} \mathrm{~s}^{-1}\right)^{-1}\right.$, assuming $1.8 \times$ $10^{20} \mathrm{~cm}^{-2}\left(\mathrm{~K} \mathrm{~km} \mathrm{~s}^{-1}\right)^{-1}$ for Milky Way clouds). In summary, Figure 12 suggests that scale of the observed structures must be considered when the total molecular mass of galaxies is calculated and that comparisons between extragalactic and Galactic molecular cloud populations should adopt the same decomposition and cloud identification methods.

\section{SUMMARY AND CONCLUSIONS}

We have presented a large-scale ${ }^{12} \mathrm{CO} J=1 \rightarrow 0$ survey of the 30 Dor molecular ridge in the LMC observed with the 
Mopra telescope. From the analysis of these observations, we conclude the following.

1. We estimate the strength of the FUV radiation field for the entire 30 Dor molecular ridge, in order to study the dependence of $\mathrm{CO}$ cloud properties on this parameter. We find that $\mathrm{CO}$ clump properties are insensitive to changes in the strength of the FUV radiation field over almost 3 orders of magnitude in $\chi$. The observed relation between CO-to$\mathrm{H}_{2}$ conversion factor and the strength of the FUV field can be reconciled with the expected increase predicted by theory if the kinetic temperature also increases in regions with strong FUV fields. Since the CO brightness temperatures do not show any significant variation along the 30 Dor molecular ridge, this would require that the angular filling factor of the $\mathrm{CO}$ emission shows a corresponding decrease with $\chi$.

2. Applying the same cloud decomposition method to clouds in the 30 Dor molecular ridge and in the outer Galaxy clouds in the 2nd Quadrant, the average CO-to- $\mathrm{H}_{2}$ conversion factor obtained in our survey is $X_{\mathrm{CO}} \simeq 2 X_{\mathrm{MW}}$. This discrepancy is smaller than has been reported by previous studies of the $\mathrm{CO}$ emission in the LMC at lower angular resolution.

3. Studying the scaling relations between the physical properties of the observed clumps, we find that the molecular clouds in the LMC ridge exhibit properties that are similar, but not identical, to those in the outer Galaxy. In particular, we find some evidence that clumps in the 30 Dor molecular ridge have larger velocity dispersions and lower brightness temperatures than their outer Galaxy counterparts. Further studies of the emission from atomic hydrogen and other carbon species in the interclump gas within LMC molecular clouds would be useful for determining the physical origin of these slight variations. The degree of virialization of the clumps does not appear to show any significant variation along the molecular ridge.

4. An analysis of the clump-mass spectra yields a powerlaw index of $\alpha=-2.0 \pm 0.4$ for the entire 30 Dor molecular ridge. The clump-mass spectrum is similar to the mass spectra obtained for Galactic clouds and for the entire LMC cloud catalog. Comparing different subregions with different average strengths of the FUV field, we find that the clump-mass spectrum is shallower in regions that are exposed to strong FUV fields. We propose that this is caused by the preferential formation of massive stars within large molecular clumps, rather than an effect of $\mathrm{CO}$ photodissociation in the low-metallicity molecular gas.

This work was supported by the Deutsche Forschungs Gemeinschaft (DFG) via Grant SFB 494. We thank Frank Israel for providing the SEST data set used to check the calibration of our data, Thomas Dame for providing the 2nd Quadrant data set, and Erik Rosolowsky for helping us with CLOUDPROPS, used to check our GAUSSCLUMPS decomposition, and with the error-invariables procedure. The National Radio Astronomy Observatory is a facility of the National Science Foundation operated under cooperative agreement by Associated Universities, Inc. We made use of the NASA/IPAC/IRAS/HiRES data reduction facilities. This research has made use of NASA's Astrophysics Data System Abstract Service.

\section{REFERENCES}

Akritas, M. G., \& Bershady, M. A. 1996, ApJ, 470, 706

Arimoto, N., Sofue, Y., \& Tsujimoto, T. 1996, PASJ, 48, 275

Aumann, H. H., Fowler, J. W., \& Melnyk, M. 1990, AJ, 99, 1674

Bakes, E. L. O., \& Tielens, A. G. G. M. 1994, ApJ, 427, 822

Blitz, L., Fukui, Y., Kawamura, A., Leroy, A., Mizuno, N., \& Rosolowsky, E. 2007, in Protostars and Planets V, ed. B. Reipurth, D. Jewitt, \& K. Keil (Tucson, AZ: Univ. Arizona Press), 81

Bolatto, A. D., Leroy, A., Israel, F. P., \& Jackson, J. M. 2003, ApJ, 595, 167

Bolatto, A. D., Leroy, A. K., Rosolowsky, E., Walter, F., \& Blitz, L. 2008, ApJ, 686, 948

Bot, C., Boulanger, F., Rubio, M., \& Rantakyro, F. 2007, A\&A, 471, 103

Brunt, C. M., Kerton, C. R., \& Pomerleau, C. 2003, ApJS, 144, 47

Cohen, R. S., Dame, T. M., Garay, G., Montani, J., Rubio, M., \& Thaddeus, P. 1988, ApJ, 331, L95

Dale, D. A., Helou, G., Contursi, A., Silbermann, N. A., \& Kolhatkar, S. 2001, ApJ, 549, 215

Dame, T. M., Elmegreen, B. G., Cohen, R. S., \& Thaddeus, P. 1986, ApJ, 305, 892

Dame, T. M., Hartmann, D., \& Thaddeus, P. 2001, ApJ, 547, 792

Dettmar, R.-J., \& Heithausen, A. 1989, ApJ, 344, L61

Dickel, J. R., McIntyre, V. J., Gruendl, R. A., \& Milne, D. K. 2005, AJ, 129, 790

Dobashi, K., Bernard, J.-P., Hughes, A., Paradis, D., Reach, W. T., \& Kawamura, A. 2008, A\&A, 484, 205

Draine, B. T. 1978 , ApJS, 36, 595

Dufour, R. J. 1984, in IAU Symp. 108, Structure and Evolution of the Magellanic Clouds, ed. S. van den Bergh \& K. S. D. Boer (Dordrecht: Kluwer), 353

Elmegreen, B. G. 1989, ApJ, 338, 178

Elmegreen, B. G., \& Falgarone, E. 1996, ApJ, 471, 816

Feast, M. 1999, PASP, 111, 775

Fukui, Y., Mizuno, N., Yamaguchi, R., Mizuno, A., \& Onishi, T. 2001, PASJ, 53, L41

Fukui, Y., et al. 1999, PASJ, 51, 745

Fukui, Y., et al. 2008, ApJS, 178, 56

Garay, G., Rubio, M., Ramirez, S., Johansson, L. E. B., \& Thaddeus, P. 1993, A\&A, 274, 743

Gaustad, J. E., McCullough, P. R., Rosing, W., \& Van Buren, D. 2001, PASP, 113,1326

Grenier, I. A., Casandjian, J.-M., \& Terrier, R. 2005, Science, 307, 1292

Habing, H. J. 1968, Bull. Astron. Inst. Neth., 19, 421

Heithausen, A., Bensch, F., Stutzki, J., Falgarone, E., \& Panis, J. F. 1998, A\&A, 331, L65

Helou, G., Khan, I. R., Malek, L., \& Boehmer, L. 1988, ApJS, 68, 151

Heyer, M., Krawczyk, C., Duval, J., \& Jackson, J. M. 2009, ApJ, 699, 1092

Heyer, M. H., \& Terebey, S. 1998, ApJ, 502, 265

Hollenbach, D. J., \& Tielens, A. G. G. M. 1999, Rev. Mod. Phys., 71, 173

Imara, N., \& Blitz, L. 2007, ApJ, 662, 969

Indebetouw, R., et al. 2008, AJ, 136, 1442

Israel, F. P. 1997, A\&A, 328, 471

Israel, F. 2000, in Molecular Hydrogen in Space, ed. F. Combes \& G. Pineau des Forêts (Cambridge: Cambridge Univ. Press), 293

Israel, F. P., Maloney, P. R., Geis, N., Herrmann, F., Madden, S. C., Poglitsch, A., \& Stacey, G. J. 1996, ApJ, 465, 738

Israel, F. P., et al. 2003, A\&A, 401, 99

Johansson, L. E. B., et al. 1998, A\&A, 331, 857

Kim, S., et al. 2007, ApJS, 171, 419

Kramer, C., Stutzki, J., Rohrig, R., \& Corneliussen, U. 1998, A\&A, 329, 249

Kramer, C., et al. 2008, A\&A, 477, 547

Kutner, M. L., et al. 1997, A\&AS, 122, 255

Ladd, N., Purcell, C., Wong, T., \& Robertson, S. 2005, PASA, 22, 62

Larson, R. B. 1981, MNRAS, 194, 809

Leroy, A., Bolatto, A., Stanimirovic, S., Mizuno, N., Israel, F., \& Bot, C. 2007, ApJ, 658, 1027

Leroy, A., Bolatto, A., Walter, F., \& Blitz, L. 2006, ApJ, 643, 825

Lisenfeld, U., \& Ferrara, A. 1998, ApJ, 496, 145

MacLaren, I., Richardson, K. M., \& Wolfendale, A. W. 1988, ApJ, 333, 821

Maloney, P., \& Black, J. H. 1988, ApJ, 325, 389

May, J., Alvarez, H., \& Bronfman, L. 1997, A\&A, 327, 325

McKee, C. F. 1989 , ApJ, 345, 782

McKee, C. F., \& Williams, J. P. 1997, ApJ, 476, 144

Meixner, M., et al. 2006, AJ, 132, 2268

Minamidani, T., et al. 2008, ApJS, 175, 485

Mizuno, N., et al. 2001a, PASJ, 53, 971

Mizuno, N., et al. 2001b, PASJ, 53, 971 
Mookerjea, B., Kramer, C., Röllig, M., \& Masur, M. 2006, A\&A, 456, 235 Myers, P. C. 1983, ApJ, 270, 105

Nakagawa, T., Yui, Y. Y., Doi, Y., Okuda, H., Shibai, H., Mochizuki, K., Nishimura, T., \& Low, F. J. 1998, ApJS, 115, 259

Ott, J., et al. 2008, PASA, 25, 129

Pak, S., Jaffe, D. T., van Dishoeck, E. F., Johansson, L. E. B., \& Booth, R. S. 1998, ApJ, 498, 735

Pineda, J. L., et al. 2008, A\&A, 482, 197

Rosolowsky, E. 2005, PASP, 117, 1403

Rosolowsky, E., \& Leroy, A. 2006, PASP, 118, 590

Rubio, M., Boulanger, F., Rantakyro, F., \& Contursi, A. 2004, A\&A, 425, L1

Rubio, M., Garay, G., Montani, J., \& Thaddeus, P. 1991, ApJ, 368, 173

Rubio, M., Lequeux, J., \& Boulanger, F. 1993, A\&A, 271, 9

Sakamoto, S. 1996, ApJ, 462, 215

Schwering, P. B. W. 1989, A\&AS, 79, 105

Sheth, K., Vogel, S. N., Wilson, C. D., \& Dame, T. M. 2008, ApJ, 675, 330

Smith, A. M., Cornett, R. H., \& Hill, R. S. 1987, ApJ, 320, 609

Solomon, P. M., Rivolo, A. R., Barrett, J., \& Yahil, A. 1987, ApJ, 319, 730
Stanimirović, S., \& Lazarian, A. 2001, ApJ, 551, L53

Stanimirović, S., Staveley-Smith, L., van der Hulst, J. M., Bontekoe, T. R., Kester, D. J. M., \& Jones, P. A. 2000, MNRAS, 315, 791

Stutzki, J., \& Güsten, R. 1990, ApJ, 356, 513

Stutzki, J., Stacey, G. J., Genzel, R., Harris, A. I., Jaffe, D. T., \& Lugten, J. B. 1988, ApJ, 332, 379

Tielens, A. G. G. M., \& Hollenbach, D. 1985, ApJ, 291, 722

van der Marel, R. P., \& Cioni, M.-R. L. 2001, AJ, 122, 1807

van Dishoeck, E. F., \& Black, J. H. 1988, ApJ, 334, 771

Verter, F., \& Hodge, P. 1995, ApJ, 446, 616

Walmsley, C. M., Natta, A., Oliva, E., \& Testi, L. 2000, A\&A, 364, 301

Weiß, A., Neininger, N., Hüttemeister, S., \& Klein, U. 2001, A\&A, 365, 571

Westerlund, B. E. 1997, The Magellanic Clouds (New York: Cambridge Univ. Press)

Williams, J. P., de Geus, E. J., \& Blitz, L. 1994, ApJ, 428, 693

Wilson, C. D. 1995, ApJ, 448, L97

Young Owl, R. C., Meixner, M. M., Wolfire, M., Tielens, A. G. G. M., \& Tauber, J. 2000, ApJ, 540, 886 